\title{
The Exact Solution of the Asymmetric Exclusion Problem With Particles of Arbitrary Size: Matrix Product Ansatz
}

\author{
Francisco C. Alcaraz and Matheus J. Lazo \\ Universidade de São Paulo, Instituto de Física de São Carlos, \\ Caixa Postal 369, 13560-590, São Carlos, SP, Brazil
}

Received on 23 April, 2003

\begin{abstract}
The exact solution of the asymmetric exclusion problem and several of its generalizations is obtained by a matrix product ansatz. Due to the similarity of the master equation and the Schrödinger equation at imaginary times the solution of these problems reduces to the diagonalization of a one dimensional quantum Hamiltonian. Initially, we present the solution of the problem when an arbitrary mixture of molecules, each of then having an arbitrary size $(s=0,1,2, \ldots)$ in units of lattice spacing, diffuses asymmetrically on the lattice. The solution of the more general problem where we have the diffusion of particles belonging to $N$ distinct classes of particles $(c=1, \ldots, N)$, with hierarchical order and arbitrary sizes, is also presented. Our matrix product ansatz asserts that the amplitudes of an arbitrary eigenfunction of the associated quantum Hamiltonian can be expressed by a product of matrices. The algebraic properties of the matrices defining the ansatz depend on the particular associated Hamiltonian. The absence of contradictions in the algebraic relations defining the algebra ensures the exact integrability of the model. In the case of particles distributed in $N>2$ classes, the associativity of this algebra implies the Yang-Baxter relations of the exact integrable model.
\end{abstract}

\section{Introduction}

The representation of interacting stochastic particle dynamics in terms of quantum spin systems produced interesting and fruitful interchanges between the areas of equilibrium and nonequilibrium statistical mechanics. The connection between these areas follows from the similarity between the master equation describing the time-fluctuations on the nonequilibrium stochastic problem and the quantum fluctuations of the equilibrium quantum spin chains [1]-[20].

Unlike the area of nonequilibrium interacting systems, where very few models are fully solvable, there exists a huge family of quantum chains appearing in equilibrium problems that are exactly integrable. The machinery that allows the exact solutions of these quantum chains comes from the Bethe ansatz in its several formulations (see [21]-[24] for reviews). The above mentioned mathematical connection between equilibrium and nonequilibrium revealed that some quantum chains related to interacting stochastic problems are exactly solvable through the Bethe ansatz. The simplest example is the problem of asymmetric diffusion of hard-core particles on the one dimensional lattice (see $[16,17,20]$ for reviews). The time fluctuations of this last model are governed by a time evolution operator that coincides with the exact integrable anisotropic Heisenberg chain, or the so called, XXZ quantum chain, in its ferromagnetically ordered regime. A generalization of this stochastic problem where exact integrability is also known [25]-[27] is the case where there are $N(N=1,2, \ldots)$ classes of particles hierarchically ordered and diffusing asymmetrically on the lattice. The quantum chain related to this problem is known in the literature as the anisotropic Sutherland model [28] or SU(3) PerkSchultz model [29]. In [15], [18] and [19] it was shown that the above mentioned asymmetric exclusion problem could also be solved exactly through the Bethe ansatz in the cases where the particles diffusing on the lattice have hard-core interactions of arbitrary range, or equivalently, the particles have size $s=0,1,2, \ldots$, in units of lattice spacing.

On the other hand, along the last decade it has been shown that the stationary distribution of probability densities of some stochastic models can also be expressed in terms of a matrix product ansatz. This means that the ground state eigenvector of the related quantum chain is also given by a matrix product ansatz. According to this ansatz the components of the ground state wavefunction are given in terms of a product of matrices. These components, apart from an overall normalization constant, are fixed by the commutation relations of the matrices defining the matrix product ansatz. These models are in general not exactly integrable [13] and the matrix product ansatz only gives the ground state wavefunction of the related Hamiltonian. Despite this limitation, this ansatz produced interesting results in a quite variety of problems including interface growth [30], boundary induced phase transitions [31]-[34], the dynamics of shocks [35] or traffic flow [36].

An interesting development of the matrix product ansatz that happened also in the area of interacting stochastic models is nowadays known as the dynamical matrix ansatz [37]. According to this new ansatz, whenever it is valid, the probability density of the stochastic system is given by a matrix 
product ansatz not only at the stationary state but at arbitrary times. In the related quantum chain this would be equivalent to the requirement that not only the ground state wavefunction, but an arbitrary one, should have its components given by a matrix product ansatz. The dynamical matrix product ansatz was applied originally to the problem of asymmetric diffusion of particles on the lattice $[37,38]$. More recently [39], [40] it has been shown that this ansatz can also be formulated in the problem of asymmetric diffusion of two types of particles. The validity of the ansatz was confirmed in the regions where the model is known to be exactly integrable through the Bethe ansatz [3, 18]. Motivated by this fact we decided to verify if we can solve the above quantum chains directly though a matrix product ansatz, without considering any time dependence as in the case of the dynamical matrix ansatz. Surprisingly, we were able to rederive all the results previously obtained though the Bethe ansatz for the asymmetric diffusion problem with one species of particles [15] or more [18, 19]. Moreover, our derivation turns out to be quite simple and it is not difficult to extend it to many other quantum Hamiltonians related or not to stochastic particle dynamics [41]. We are going to present in this paper these derivations and, as we shall see, many of the results obtained in [15], [18] and [19] can now be rederived quite easily. The simplicity of our ansatz enabled us to extend the results of [19] to the case where each individual particle $i$ belonging to any class $(c=1, \ldots, N)$ is distinguishable with a given size $s_{i}\left(s_{i}=0,1, \ldots\right)$.

This paper is organized as follows. In the next section we review the asymmetric diffusion problem with a single type of particles of arbitrary size and we derive the associated quantum chain. In section 3 we introduce the matrix product ansatz and obtain the exact solution of the model presented in section 2. Similarly as in section 2 , in section 4 we derive the quantum Hamiltonian associated with the problem of asymmetric diffusion of several types of particles with arbitrary sizes and hierarchical order. In section 5 the exact solution of the general model of section 4 is obtained though an appropriate matrix product ansatz. Finally in section 6 we conclude our paper with some final comments and conclusions.

\section{The asymmetric exclusion model with particles of arbitrary sizes}

The standard asymmetric exclusion model is a onedimensional stochastic model that describes the time fluctuations of hard-core particles diffusing asymmetrically on the lattice. If we denote an occupied site $i$ on the lattice by $\sigma_{i}^{z}=+1$ and a vacant site $i$ by $\sigma_{i}^{z}=-1$, the time evolution operator of the probability distribution of particles is given by the asymmetric XXZ Hamiltonian,

$$
H=-\sum_{i=1}^{L}\left[\epsilon_{+} \sigma_{i}^{-} \sigma_{i+1}^{+}+\epsilon_{-} \sigma_{i}^{+} \sigma_{i+1}^{-}+\frac{1}{4}\left(1-\sigma_{i}^{z} \sigma_{i+1}^{z}\right)\right],
$$

where $L$ is the number of lattice sizes and $\sigma^{ \pm}=$ $\left(\sigma^{x} \pm i \sigma^{y}\right) / 2$ are the raising and lowering spin $-1 / 2$ Pauli operators. Periodic boundary conditions are imposed and $\epsilon_{+}$ and $\epsilon_{-}\left(\epsilon_{+}+\epsilon_{-}=1\right)$ are the transition probabilities for the motions to the right and left, respectively. It is important to notice that this Hamiltonian, contrary to the standard XXZ quantum chain, is not Hermitian for $\epsilon_{+} \neq \epsilon_{-}$. Such property, besides producing complex eigenvalues, also produces massless regime, in a region where the standard $\mathrm{XXZ}$ is massive (gapped), whose mass gap vanishes as $L^{-3 / 2}[10]$ [12].

The generalization of this problem, that we consider in this section, is obtained by considering that each distinct particle, instead of having an excluded hard-core volume of a single lattice size $(s=1)$, may now have a hard-core volume of $s$ sites $(s=0,1,2, \ldots)$. Equivalently, each individual particle on the lattice may have a distinct size $s=0,1,2, \ldots$. Particles of sizes $s$ on the lattice are composed by $s$ onesite monomers and we represent their coordinates by giving the position of their leftmost monomer. In Fig. 1 some examples were shown for the configurations with $n=5$ molecules and some size distributions $\{s\}$ in a lattice with $L=5$ sites. We should notice that molecules of size $s=0$ have no excluded volume interaction and we can have an arbitrary number of them in a given site. However, we should stress that although being sizeless they keep the order of the size distribution on the lattice. This means that if a given particle of size $s$ is initially between particles of size $s^{\prime}$ and $s^{\prime \prime}$ it will keep this relative order in future times.

a)

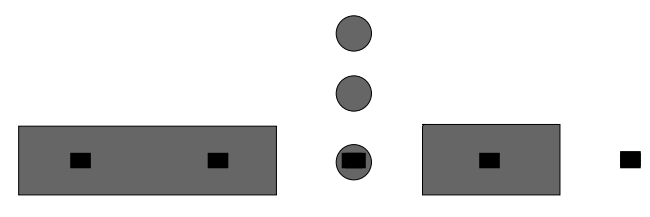

$\{s\}=\{2,0,0,0,1\}$

b)

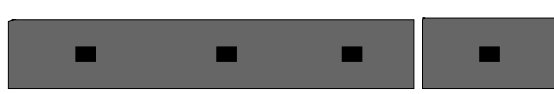

$\{\mathrm{s}\}=\{3,1,0,0,0\}$

c)
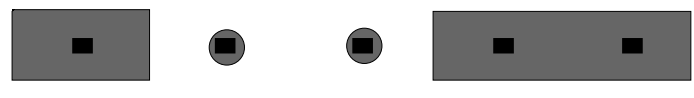

$\{s\}=\{1,0,0,0,2\}$

Figure 1. Example of configurations of molecules with distinct sizes $s$ in a lattice of size $L=5$.

In order to describe the occupancy of a given site $i$ $(1,2, \ldots, N)$ we attach on it a site variable $\beta_{i}$ taking integer values $\left(\beta_{i} \in \mathbf{Z}\right)$. If $\beta_{i}=0$, the site is vacant (or may be occupied by a monomer of the molecule on its leftmost neighboring site). If $\beta_{i}>0$, we have on the site a molecule of size $s=\beta_{i}$ and the sites $j=i+1, \ldots, i+\beta_{i}-1$ are empty sites. Finally, if $\beta_{i}=-n<0$, we have, at the site $i, n$ molecules of size zero. The allowed configurations, denoted by $\left\{\beta_{i}\right\}=\left\{\beta_{1}, \beta_{2}, \ldots, \beta_{N}\right\}$, are those satisfying the hard-core constraints imposed by the sizes of the molecules on the periodic lattice. This means that if in a given configuration $\left\{\beta_{i}\right\}$ we have $\beta_{j} \neq 0$ and $\beta_{l} \neq 0$ then we should have $l-j \geq \beta_{l}$ or $j-l \geq \beta_{j}$, depending if $l>j$ or $l<j$, respectively (see Fig. 1). 
The master equation for the probability distribution at a given time $t, P(\{\beta\}, t)$, can be written in general as

$$
\frac{\partial P(\{\beta\}, t)}{\partial t}=-\Gamma\left(\{\beta\} \rightarrow\left\{\beta^{\prime}\right\}\right) P(\{\beta\}, t)+\Gamma\left(\left\{\beta^{\prime}\right\} \rightarrow\{\beta\}\right) P\left(\left\{\beta^{\prime}\right\}, t\right),
$$

where $\Gamma\left(\{\beta\} \rightarrow\left\{\beta^{\prime}\right\}\right)$ is the transition rate where a configuration $\{\beta\}$ changes to $\left\{\beta^{\prime}\right\}$. In the model we are considering there exists only diffusion processes. The allowed motions, whenever there is no hard-core constraints, are those in which a given particle diffuses to its right,

$$
\begin{aligned}
& \beta_{i} \emptyset_{i+1} \rightarrow \emptyset_{i} \beta_{i+1}, \beta>0, \\
& \beta_{i} \gamma_{i+1} \rightarrow(\beta+1)_{i}(\gamma-1)_{i+1}, \beta<0, \gamma \leq 0,(3)
\end{aligned}
$$

with transition rate $\epsilon_{R}$, and diffuses to the left,

$$
\begin{aligned}
& \emptyset_{i} \beta_{i+1} \rightarrow \beta_{i} \emptyset_{i+1}, \quad \beta>0, \\
& \gamma_{i} \beta_{i+1} \rightarrow(\gamma-1)_{i}(\beta+1)_{i+1}, \beta<0, \gamma \leq 0,(4)
\end{aligned}
$$

with transition rate $\epsilon_{L}$. The master equation (2) can be written as a Schrödinger equation in Euclidean time (see [3] for general applications for two-body processes),

$$
\frac{\partial|P\rangle}{\partial t}=-H|P\rangle
$$

if we interpret $|P\rangle \equiv P(\{\beta\}, t)$ as the associated wave function. If we represent $\beta_{i}$ as $|\beta\rangle_{i}$, the vectors $|\beta\rangle_{1} \otimes|\beta\rangle_{2} \otimes$ $\cdots \otimes|\beta\rangle_{N}$ will span the associated Hilbert space. The diffusion process given in (3) and (4) is associated with the Hamiltonian [3]

$$
\begin{aligned}
H & =-D \mathcal{P} \sum_{i=1}^{L}\left(H_{i}^{>}+H_{i}^{<}\right) \mathcal{P}, \\
H_{i}^{>} & =\sum_{\beta=1}^{\infty}\left[\epsilon_{+}\left(1-E_{i}^{\beta, 0} E_{i+1}^{0, \beta}\right) \mathcal{P} E_{i}^{0, \beta} E_{i+1}^{\beta, 0}+\epsilon_{-}\left(1-E_{i}^{0, \beta} E_{i+1}^{\beta, 0}\right) \mathcal{P} E_{i}^{\beta, 0} E_{i+1}^{0, \beta}\right], \\
H_{i}^{<} & =\sum_{\beta=-\infty}^{-1} \sum_{\gamma=-\infty}^{0}\left[\epsilon_{+}\left(E_{i}^{\beta+1, \beta} E_{i+1}^{\gamma-1, \gamma}-E_{i}^{\beta, \beta} E_{i+1}^{\gamma, \gamma}\right)+\epsilon_{-}\left(E_{i}^{\gamma-1, \gamma} E_{i+1}^{\beta+1, \beta}-E_{i}^{\gamma, \gamma} E_{i+1}^{\beta, \beta}\right)\right],
\end{aligned}
$$

with

$$
D=\epsilon_{R}+\epsilon_{L}, \quad \epsilon_{+}=\frac{\epsilon_{R}}{\epsilon_{R}+\epsilon_{L}}, \quad \epsilon_{-}=\frac{\epsilon_{L}}{\epsilon_{R}+\epsilon_{L}},
$$

and periodic boundary conditions. The matrices $E^{\alpha, \beta}$ are infinite-dimensional with a single nonzero element $\left(E^{\alpha, \beta}\right)_{i, j}=$ $\delta_{\alpha, i} \delta_{\beta, j}(\alpha, \beta, i, j \in \mathbf{Z})$. The projector $\mathcal{P}$ projects out the configurations $|\{\beta\}\rangle>$ satisfying the constraint that for all $\beta_{i}, \beta_{j} \neq$ $0:(j-i) \geq s_{i}$ if $j>i$ or $(i-j) \geq s_{j}$ if $i>j$. The constant $D$ in (6) fixes the time scale, and for simplicity we chose $D=1$. A simplification of our general problem happens when all the particles have the same size $s>0$. In this case the matrices $E^{\alpha, \beta}$ can be replaced by the spin-1/2 Pauli matrices and the Hamiltonian is given by

$$
H_{\left\{s_{1}=\cdots=s_{n}=s\right\}}=-\mathcal{P}_{s}\left(\sum_{i=1}^{L}\left[\epsilon_{+} \sigma_{i}^{-} \sigma_{i+1}^{+}+\epsilon_{-} \sigma_{i}^{+} \sigma_{i+1}^{-}\right]+\frac{1}{4}\left(\epsilon_{+}+\epsilon_{-}\right)\left(\sigma_{i}^{z} \sigma_{i+s}^{z}-1\right)\right) \mathcal{P}_{s},
$$

where now $\mathcal{P}_{s}$ projects out the configuration where two up spins, in the $\sigma^{z}$-basis, are at distance smaller than the size $s>0$ of the particles. The simplest case $s=1$ gives $\mathcal{P}_{s}=1$ and we obtain the standard asymmetric exclusion Hamiltonian (1). For the sake of comparison with the standard XXZ chain, normally considered in the context of magnetic systems, for $\epsilon_{+}, \epsilon_{-} \neq 0$, we perform the canonical transformation:

$$
\sigma_{i}^{ \pm} \rightarrow\left(\frac{\epsilon_{-}}{\epsilon_{+}}\right)^{ \pm \frac{i}{2}} \sigma_{i}^{ \pm}, \sigma^{z} \rightarrow \sigma^{z}, \quad(i=0,1,2, \ldots, L)
$$

in Eq. (8) and obtain

$$
\begin{aligned}
H & =-\frac{1}{2} \sqrt{\epsilon_{+} \epsilon_{-}} \sum_{i=1}^{L} \mathcal{P}_{s}\left[\sigma_{i}^{x} \sigma_{i+1}^{x}+\sigma_{i}^{y} \sigma_{i+1}^{y}+\Delta\left(\sigma_{i}^{z} \sigma_{i+s}^{z}-1\right)\right] \mathcal{P}_{s}, \\
\Delta & =\frac{\epsilon_{+}+\epsilon_{-}}{2 \sqrt{\epsilon_{+} \epsilon_{-}}} .
\end{aligned}
$$


Apart from the projector, this Hamiltonian coincides with the gapped ferromagnetic Heisenberg chain. However now, in distinction with (8), the boundary condition is not periodic but twisted,

$$
\sigma_{N+1}^{ \pm}=\left(\frac{\epsilon_{+}}{\epsilon_{-}}\right)^{ \pm \frac{L}{2}} \sigma_{1}^{ \pm}, \sigma_{N+1}^{z}=\sigma_{1}^{z} .
$$

Since $\epsilon_{+} / \epsilon_{-} \neq 1$ this boundary term has the same degree of importance as the whole system, and we have a critical behavior induced by the surface, i. e., the mass gap vanishes in opposition to the standard periodic ferromagnetic XXZ chain.

\section{The exact solution of the gener- alized asymmetric exclusion prob- lem: The matrix product ansatz}

The exact solution of the generalized asymmetric exclusion problem of last section was derived in [15] within the frame- work of the coordinate Bethe ansatz. In this section we are going to rederive this solution by imposing a matrix product ansatz for the eigenfunctions of the Hamiltonian (6). As we shall see, this derivation turns out to be more direct than the old one presented in [15].

Before considering the more general situation where any molecule may have a distinct size let us consider initially the simple case where all the molecules have the same size $s(s=0,1, \ldots)$.

Since the diffusion process conserves particles, and the lattice is periodic, the total number of particles $n$ and the momentum $P$ are good quantum numbers. Consequently the Hilbert space associated with (6) can be separated into block disjoint sectors labelled by the values of $n(n=0,1, \ldots)$ and $P(P=2 \pi l / L ; l=0,12, \ldots, L-1)$.

Our ansatz asserts that any eigenfunction $\left|\Psi_{n, P}\right\rangle$ of (6) in the sector with $n$ particles and momentum $P$ will have its components given by the matrix product

$$
\begin{aligned}
\left|\Psi_{n, P}\right\rangle & =\sum_{\left\{x_{1}, \ldots, x_{n}\right\}}^{*} f\left(x_{1}, \ldots, x_{n}\right)\left|x_{1}, \ldots, x_{n}\right\rangle, \\
f\left(x_{1}, \ldots, x_{n}\right) & =\operatorname{Tr}\left[E^{x_{1}-1} A^{(s)} E^{x_{2}-x_{1}-1} A^{(s)} \cdots E^{x_{n}-x_{n-1}-1} A^{(s)} E^{L-x_{n}} \Omega_{P}\right] .
\end{aligned}
$$

The ket $\left|x_{1}, \ldots, x_{n}\right\rangle$ denotes the configuration where the particles are located at $\left(x_{1}, \ldots, x_{n}\right)$, and the symbol (*) in the sum denotes the restriction to the sets satisfying the hard-core exclusion due to the size $s$ of the particles, i. e.,

$$
x_{i+1} \geq x_{i}+s, \quad i=1, \ldots, n-1, \quad s \leq x_{n}-x_{1} \leq L-s,
$$

where we have to remember that in the case where the particles have size $s=0$ we may have any number of particle in a given site. Differently from the standard Bethe ansatz, in which $f\left(x_{1}, \ldots, x_{n}\right)$ is given by a combination of plane waves, now it is given by the trace of a product of matrices. The matrices $E$ and $A^{(s)}$ are associated with the empty and occupied sites describing the configuration of the lattice. The superscript $(s)$ is just to remember the size of the particle. The matrix $\Omega_{P}$ in (12) is introduced in order to ensure the momentum $P$ of the eigenfunction $\left|\Psi_{n, P}\right\rangle$. This is accomplished by imposing the commutation relation

$$
E \Omega_{P}=e^{-i P} \Omega_{P} E, \quad A^{(s)} \Omega_{P}=e^{-i P} \Omega_{P} A^{(s)},
$$

since, from (12), we must have for eigenfunctions of momentum $P$ the ratio of the amplitudes

$$
\frac{f\left(x_{1}, \ldots, x_{n}\right)}{f\left(x_{1}+m, \ldots, x_{n}+m\right)}=e^{-i m P}, \quad(m=0,1,2, \ldots, L-1) .
$$

The algebraic properties of $A^{(s)}$ and $E$ will be fixed by requiring that $\left|\Psi_{n, P}\right\rangle$, defining the ansatz (12), satisfy the eigenvalue equation

$$
H_{\left\{s_{1}=\cdots=s_{n}=s\right\}}\left|\Psi_{n, P}\right\rangle=\varepsilon_{n}\left|\Psi_{n, P}\right\rangle,
$$

where $H_{\left\{s_{1}=\cdots=s_{n}=s\right\}}$ is given by (8).

Before considering the case where $n$ is general, let us consider the cases where we have only $n=1$ or $n=2$ particles.

$\mathbf{n}=\mathbf{1}$. For one particle the eigenvalue equation (16) gives

$$
\begin{aligned}
\varepsilon_{1} \operatorname{Tr}\left(E^{x_{1}-1} A^{(s)} E^{L-x_{1}} \Omega_{P}\right) & =-\epsilon_{+} \operatorname{Tr}\left(E^{x_{1}-2} A^{(s)} E^{L-x_{1}+1} \Omega_{P}\right) \\
-\epsilon_{-} \operatorname{Tr}\left(E^{x_{1}} A^{(s)} E^{L-x_{1}-1} \Omega_{P}\right) & +\left(\epsilon_{+}+\epsilon_{-}\right) \operatorname{Tr}\left(E^{x_{1}-1} A^{(s)} E^{L-x_{1}} \Omega_{P}\right) .
\end{aligned}
$$


The cyclic property of the trace and the algebra (14) fix the values of the energies,

$$
\varepsilon_{1}=-\left(\epsilon_{+} e^{-i P}+\epsilon_{-} e^{i P}-1\right)
$$

where $P=\frac{2 \pi l}{L}(l=0,1, \ldots, L-1)$ is the momentum of the state.

An alternative way to solve (17) that will be easier to generalize for arbitrary values of $n$ is obtained by the replacement

$$
A^{(s)}=A_{k}^{(s)} E^{2-s},
$$

where $A_{k}$ is a spectral parameter dependent matrix with the following commutation relation with the matrix $E$,

$$
E A_{k}^{(s)}=e^{i k} A_{k}^{(s)} E .
$$

Inserting (19) in (17) and using (20) we obtain

$$
\varepsilon_{1}=\varepsilon(k)=-\left(\epsilon_{+} e^{-i k}+\epsilon_{-} e^{i k}-1\right),
$$

where we have used $\epsilon_{+}+\epsilon_{-}=1$.

Comparing (21) with (18) we fix the spectral parameter $k$ as the momentum of the 1-particle eigenfunction $\left|\Psi_{1, P}\right\rangle$, i. e., $k=P=\frac{2 \pi l}{L}(l=0,1, \ldots, L-1)$.

$\mathbf{n}=2$. For two particles on the lattice the eigenvalue equation (16) gives for $\left|\Psi_{2, P}\right\rangle$ two types of relations depending on the relative location of the particles. The amplitudes corresponding to the configuration $\left|x_{1}, x_{2}\right\rangle$ where $x_{2}>x_{1}+s$ will give the relation

$$
\begin{aligned}
\varepsilon_{2} \operatorname{Tr}\left(E^{x_{1}-1} A^{(s)} E^{x_{2}-x_{1}-1} A^{(s)} E^{L-x_{2}} \Omega_{P}\right) & =-\epsilon_{+} \operatorname{Tr}\left(E^{x_{1}-2} A^{(s)} E^{x_{2}-x_{1}} A^{(s)} E^{L-x_{2}} \Omega_{P}\right) \\
-\epsilon_{-} \operatorname{Tr}\left(E^{x_{1}} A^{(s)} E^{x_{2}-x_{1}-2} A^{(s)} E^{L-x_{2}} \Omega_{P}\right) & -\epsilon_{+} \operatorname{Tr}\left(E^{x_{1}-1} A^{(s)} E^{x_{2}-x_{1}-2} A^{(s)} E^{L-x_{2}+1} \Omega_{P}\right) \\
-\epsilon_{-} \operatorname{Tr}\left(E^{x_{1}-1} A^{(s)} E^{x_{2}-x_{1}} A^{(s)} E^{L-x_{2}-1} \Omega_{P}\right) & +2 \operatorname{Tr}\left(E^{x_{1}-1} A^{(s)} E^{x_{2}-x_{1}-1} A^{(s)} E^{L-x_{2}} \Omega_{P}\right) .
\end{aligned}
$$

A possible and convenient way to solve this equation is by identifying the matrices $A^{(s)}$ as composed by two spectral parameter-dependent new matrices $A_{k_{1}}^{(s)}$ and $A_{k_{2}}^{(s)}$,i. e.,

$$
A^{(s)}=\sum_{i=1}^{2} A_{k_{i}}^{(s)} E^{2-s},
$$

that satisfy, as in (20), the commutation relation

$$
E A_{k_{j}}^{(s)}=e^{i k_{j}} A_{k_{j}}^{(s)} E, \quad(j=1,2) .
$$

Inserting (23) in (22) and using (24) we obtain

$$
\varepsilon_{2}=\varepsilon\left(k_{1}\right)+\varepsilon\left(k_{2}\right),
$$

where $\epsilon(k)$ is given in (21).
The relation (14) gives the commutation of these new matrices $A_{k_{i}}^{(s)}$ with $\Omega_{P}$, i. e.,

$$
A_{k_{j}}^{(s)} \Omega_{P}=e^{i P(1-s)} \Omega_{P} A_{k_{j}}^{(s)}, \quad(j=1,2) .
$$

Comparing the components of the configurations $\left|x_{1}, x_{2}\right\rangle$ and $\left|x_{1}+m, x_{2}+m\right\rangle$, and exploring the cyclic invariance of the trace, we obtain

$$
P=k_{1}+k_{2}
$$

Up to now the commutation relations of the matrices $A_{k_{1}}^{(s)}$ and $A_{k_{2}}^{(s)}$ among themselves as well the spectral parameters, that in general may be complex, are unknown. The eigenvalue equation (16) when applied to the components of the configuration $\left|x_{1}, x_{2}\right\rangle$ where $x_{2}=x_{1}+s$ ("matching" conditions) will give us the relation

$$
\begin{aligned}
\varepsilon_{2} \operatorname{Tr}\left(E^{x_{1}-1} A^{(s)} E^{s-1} A^{(s)} E^{L-x_{1}-s} \Omega_{P}\right) & =-\epsilon_{+} \operatorname{Tr}\left(E^{x_{1}-2} A^{(s)} E^{s} A^{(s)} E^{L-x_{1}-s} \Omega_{P}\right) \\
-\epsilon_{-} \operatorname{Tr}\left(E^{x_{1}-1} A^{(s)} E^{s} A^{(s)} E^{L-x_{1}-s-1} \Omega_{P}\right) & +\operatorname{Tr}\left(E^{x_{1}-1} A^{(s)} E^{s-1} A^{(s)} E^{L-x_{1}-s} \Omega_{P}\right) .
\end{aligned}
$$

Using (23) to express the $A^{(s)}$ matrix in terms of the spectral parameter matrices $A_{k_{j}}^{(s)}(j=1,2)$, and (21) for $\varepsilon_{2}$, the last expression gives

$$
\sum_{j, l}^{2}\left[\epsilon_{-}-e^{-i k_{j}}+\epsilon_{+} e^{-i\left(k_{j}+k_{l}\right)}\right] A_{k_{j}}^{(s)} A_{k_{l}}^{(s)}=0 .
$$


This last relation implies that the matrices $\left\{A_{k_{j}}\right\}$ should obey the algebra

$$
A_{k_{j}}^{(s)} A_{k_{l}}^{(s)}=S\left(k_{j}, k_{l}\right) A_{k_{l}}^{(s)} A_{k_{j}}^{(s)}, \quad(l \neq j), \quad\left(A_{k_{j}}^{(s)}\right)^{2}=0,
$$

where $(l, j)=1,2$ and

$$
S\left(k_{j}, k_{l}\right)=-\frac{\epsilon_{+}+\epsilon_{-} e^{i\left(k_{j}+k_{l}\right)}-e^{i k_{j}}}{\epsilon_{+}+\epsilon_{-} e^{i\left(k_{j}+k_{l}\right)}-e^{i k_{l}}} .
$$

$$
\begin{aligned}
\operatorname{Tr}\left(A_{k_{l}}^{(s)} A_{k_{j}}^{(s)} E^{L-2 s+2} \Omega_{P}\right) & =e^{-i(L-2 s+2) k_{j}} \operatorname{Tr}\left(A_{k_{l}}^{(s)} E^{L-2 s+2} A_{k_{j}}^{(s)} \Omega_{P}\right) \\
& =e^{-i k_{j} L} e^{i 2 k_{j}(s-1)} e^{-i P(s-1)} \operatorname{Tr}\left(A_{k_{j}}^{(s)} A_{k_{l}}^{(s)} E^{L-2 s+2} \Omega_{P}\right) \\
& =e^{-i k_{j} L} e^{i 2 k_{j}(s-1)} e^{-i P(s-1)} S\left(k_{j}, k_{l}\right) \operatorname{Tr}\left(A_{k_{l}}^{(s)} A_{k_{j}}^{(s)} E^{L-2 s+2} \Omega_{P}\right),
\end{aligned}
$$

or equivalently, since $P=k_{1}+k_{2}$,

$$
e^{i k_{j} L}=S\left(k_{j}, k_{l}\right)\left(\frac{e^{i k_{j}}}{e^{i k_{l}}}\right)^{s-1}, \quad j=1,2 \quad(j \neq l) .
$$

The energy and momentum are given by inserting the solution of (33) into (25) and (27), respectively.
The complex spectral parameters $\left\{k_{j}\right\}$ are still free up to now. The cyclic property of the trace together with the algebraic relations (14), (24), (26) and (30) and the fact that any component should be uniquely related give us

tion of (33) into (25) and (27), respectively.

$$
\begin{gathered}
\varepsilon_{n} \operatorname{Tr}\left(\cdots E^{x_{i}-x_{i-1}-1} A^{(s)} E^{x_{i+1}-x_{i}-1} A^{(s)} \cdots A^{(s)} E^{L-x_{n}} \Omega_{P}\right)= \\
-\sum_{i=1}^{n}\left\{\epsilon_{+} \operatorname{Tr}\left(\cdots E^{x_{i}-x_{i-1}-2} A^{(s)} E^{x_{i+1}-x_{i}} A^{(s)} \cdots A^{(s)} E^{L-x_{n}} \Omega_{P}\right)\right. \\
+\epsilon_{-} \operatorname{Tr}\left(\cdots E^{x_{i}-x_{i-1}-1} A^{(s)} E^{x_{i+1}-x_{i}-2} A^{(s)} \cdots A^{(s)} E^{L-x_{n}+1} \Omega_{P}\right) \\
\left.-\operatorname{Tr}\left(\cdots E^{x_{i}-x_{i-1}-1} A^{(s)} E^{x_{i+i}-x_{i}-1} A^{(s)} \cdots A^{(s)} E^{L-x_{n}} \Omega_{P}\right)\right\} .
\end{gathered}
$$

The solution is obtained by identifying the $A^{(s)}$ matrix as a combination of $n$ spectral parameter-dependent $\left\{A_{k_{j}}^{(s)}\right\}$ matrices, namely,

$$
A^{(s)}=\sum_{j=1}^{n} A_{k_{j}}^{(s)} E^{2-s}
$$

with the commutation relations with the matrices $E$ and $\Omega_{P}$,

$$
\begin{gathered}
E A_{k_{j}}^{(s)}=e^{i k_{j}} A_{k_{j}}^{(s)} E \\
A_{k_{j}}^{(s)} \Omega_{P}=e^{i P(1-s)} \Omega_{P} A_{k_{j}}^{(s)}(j=1, \ldots, n) .
\end{gathered}
$$

Inserting (35) into (34) and using the relations (36), together with the cyclic property of the trace, we obtain

$$
\varepsilon_{n}=\sum_{j=1}^{n} \varepsilon\left(k_{j}\right), \quad P=\sum_{j=1}^{n} k_{j},
$$

for the energy and momentum of $\left|\Psi_{n, P}\right\rangle$, respectively. The eigenvalue equation (16) applied to the configuration where a pair of particles located at $x_{i}$ and $x_{i+1}$ are at the closest position, i. e., $x_{i+1}=x_{i}+s$, will give relations that coincide with (30) and (31), but now with $j=1,2, \ldots, n$. The configurations of $\left|\Psi_{n, P}\right\rangle$ corresponding to three or more particles at the "matching" distances will demand that the algebra satisfied by the matrices $\left\{A_{k_{j}}\right\}$ in (30) is associative. Equivalently, this means that a given component, expressed in terms of a product of matrices $\left\{A_{k_{j}}\right\}$ and $E$, should be uniquely related to the other components. This is imme- 
diate for the present problem since the structure constants $S\left(k_{j}, k_{l}\right)$ of the algebra in (30) are constants with the property

$$
S\left(k_{j}, k_{l}\right) S\left(k_{l}, k_{j}\right)=1 .
$$

As we are going to see in section 4, this condition in general leads to the well-known Yang-Baxter relations [42, 21].

Once the algebra is defined all the components of $\left|\Psi_{n, P}\right\rangle$ can be uniquely determined only if this algebra has a well defined trace, whose cyclic property will fix the $n$ complex spectral parameters $\left\{k_{j}\right\}$. An analog procedure as in (32) gives us the constraints

$$
e^{i k_{j} L}=(-1)^{n} \prod_{l=1}^{n}\left(\frac{e^{i k_{j}}}{e^{i k_{l}}}\right)^{s-1} \frac{\epsilon_{+}+\epsilon_{-} e^{i\left(k_{j}+k_{l}\right)}-e^{i k_{j}}}{\epsilon_{+}+\epsilon_{-} e^{i\left(k_{j}+k_{l}\right)}-e^{i k_{l}}} .
$$

This equation coincides with the Bethe-ansatz equations derived in [15] through the coordinate Bethe ansatz method. Moreover, an arbitrary component $f\left(x_{1}, \ldots, x_{n}\right)$ of the wave function $\left|\Psi_{n, P}\right\rangle$ given in (12) can be written as

$$
\begin{aligned}
& f\left(x_{1}, \ldots, x_{n}\right)= \\
& \sum_{i_{1}=1}^{n} \sum_{i_{2}=1}^{n} \cdots \sum_{i_{n}=1}^{n} \operatorname{Tr}\left(E^{x_{1}-1} A_{k_{i_{1}}}^{(s)} E^{x_{2}-x_{1}+1-s} A_{k_{i_{2}}}^{(s)} \cdots E^{x_{n}-x_{n-1}+1-s} A_{k_{i_{n}}}^{(s)} E^{L-x_{n}+2-s} \Omega_{P}\right) .
\end{aligned}
$$

Using the commutation relation (24) and the fact that $\left(A_{k_{j}}^{(s)}\right)^{2}=0(j=1, \ldots, n)$, we can rewrite this last expression as

$$
\begin{aligned}
& f\left(x_{1}, \ldots, x_{n}\right)= \\
& \sum_{i_{1}, \ldots, i_{n}} e^{i\left[k_{i_{1}}\left(x_{1}-1\right)+k_{i_{2}}\left(x_{2}-1\right)+\cdots+k_{i_{n}}\left(x_{n}-1\right)\right]} \operatorname{Tr}\left(A_{k_{i_{1}}}^{(s)} E^{1-s} A_{k_{i_{2}}}^{(s)} E^{1-s} \cdots E^{1-s} A_{k_{i_{n}}}^{(s)} E^{L} \Omega_{P}\right) .
\end{aligned}
$$

Let us define the new matrices

$$
\tilde{A}_{k_{j}}^{(s)}=A_{k_{j}}^{(s)} E^{1-s} \quad(j=1, \ldots, n) .
$$

It is simple to verify, from (36), that they satisfy

$$
\tilde{A}_{k_{j}}^{(s)} \tilde{A}_{k_{l}}^{(s)}=\tilde{S}\left(k_{j}, k_{l}\right) \tilde{A}_{k_{l}}^{(s)} \tilde{A}_{k_{j}}^{(s)}, \quad(j \neq l), \quad\left(\tilde{A}_{k_{j}}^{(s)}\right)^{2}=0,
$$

where

$$
\tilde{S}\left(k_{j}, k_{l}\right)=S\left(k_{j}, k_{l}\right)\left(\frac{e^{i k_{j}}}{e^{i k_{l}}}\right)^{s-1} .
$$

Finally, in terms of these new matrices, and exploring the fact that $\left(\tilde{A}_{k_{j}}^{(s)}\right)^{2}=0$, we can write

$$
f\left(x_{1}, \ldots, x_{n}\right)=\sum_{p_{1}, \ldots, p_{n}} e^{i\left[k_{p_{1}}\left(x_{1}-1\right)+k_{p_{2}}\left(x_{2}-1\right)+\cdots+k_{p_{n}}\left(x_{n}-1\right)\right]} \operatorname{Tr}\left(\tilde{A}_{k_{p_{1}}}^{(s)} \tilde{A}_{k_{p_{2}}}^{(s)} \cdots \tilde{A}_{k_{p_{n}}}^{(s)} E^{L} \Omega_{P}\right),
$$

where the sum is over the permutations $\left(p_{1}, p_{2}, \ldots, p_{n}\right)$ of non repeated integers $(1,2, \ldots, n)$. The result (45) shows us that the amplitudes derived using the present matrix product ansatz are given by a combination of plane waves with complex wave number $\left\{k_{j}\right\}$, and reproduce the results obtained previously [15] through the standard coordinate Bethe ansatz.

Let us return to the general case where we have $n$ molecules with arbitrary sizes $\left\{s_{1}, s_{2}, \ldots, s_{n}\right\}$ and whose related Hamiltonian is given by (6). In this general case each particle is conserved separately, and since in the diffusion processes no interchange of particles are allowed, also the order $\left\{s_{1}, s_{2}, \ldots, s_{n}\right\}$ where the particles appear is a constant of motion, up to cyclic permutations. The eigenfunction corresponding to a given order $\left\{s_{1}, s_{2}, \ldots, s_{n}\right\}$ and momentum $P$ can be written as

$$
\left|\Psi_{\left\{s_{1}, \ldots, s_{n}\right\}, P}\right\rangle=\sum_{\{c\}} \sum_{\{x\}} f^{s_{c_{1}}, \ldots, s_{c_{n}}}\left(x_{1}, \ldots, x_{n}\right)\left|x_{1}, \ldots, x_{n}\right\rangle,
$$


where $f^{s_{c_{1}}, \ldots, s_{c_{n}}}\left(x_{1}, \ldots, x_{n}\right)$ is the component of a configuration where the particles of sizes $s_{c_{1}}, \ldots, s_{c_{n}}$ are located at positions $x_{1}, \ldots, x_{n}$ respectively. The summation $\{c\}$ extends over all cyclic permutations $\left\{c_{1}, \ldots, c_{n}\right\}$ of integers $\{1, \ldots, n\}$, and the summation $\{x\}$ extends, for a given distribution $\left\{s_{c_{1}}, \ldots, s_{c_{n}}\right\}$ of molecules, to increasing integers satisfying

$$
\begin{array}{r}
x_{i+1}-x_{i} \geq s_{c_{i}}, \quad i=1, \ldots, n-1 \\
s_{c_{1}} \leq x_{n}-x_{1} \leq N-s_{c_{n}} .
\end{array}
$$

In order to formulate our matrix product ansatz we associate with the sites occupied by the particles of size $s_{j}$ $(j=1, \ldots, n)$ a matrix $A^{\left(s_{j}\right)}$ and with the remaining $L-n$ sites we associate, as before, the matrix $E$. Our ansatz asserts, in a generalization of (12), that the amplitudes of the eigenfunctions (46) are given by

$$
f^{s_{1}, \ldots, s_{n}}\left(x_{1}, \ldots, x_{n}\right)=\operatorname{Tr}\left(E^{x_{1}-1} A^{\left(s_{1}\right)} E^{x_{2}-x_{1}-1} A^{\left(s_{2}\right)} \cdots E^{x_{n}-x_{n-1}-1} A^{\left(s_{n}\right)} E^{L-x_{n}} \Omega_{P}\right)
$$

where in order to ensure the momentum $P$ of the eigenstate the matrices $\left\{A^{\{s\}}\right\}$ should satisfy

$$
E \Omega_{P}=e^{-i P} \Omega_{P} E, \quad A^{(s)} \Omega_{P}=e^{-i P} \Omega_{P} A^{(s)} .
$$

Let us initially consider our ansatz (46)-(49) for $n=1$ and $n=2$ molecules.

\section{$\mathbf{n}=\mathbf{1}$}

For one particle on the chain we have the same ansatz as (12) and the energy given by (18).

$\mathbf{n}=\mathbf{2}$.

In this case, if both particles have the same sizes $s_{1}=$ $s_{2}=s$, we have the same situation considered previously in (22)-(33). The eigenfunctions $\left|\Psi_{\{s, s\}, P}\right\rangle$ will be given by (12) and the energy by (24) with $k_{i}$ fixed by (33).

If the particles are distinct, the situation is new. The eigenvalue equation when applied to the configurations where the two particles of sizes $s_{1}$ and $s_{2}$ are located at $x_{1}$ and $x_{2} \geq x_{1}+s_{1}$, respectively, will give, mutatis mutandis, an expression similar to (32). The corresponding situation is obtained by introducing the generalization of the spectral parameter matrices defined on (23), i. e.,

$$
A^{\left(s_{j}\right)}=\sum_{l=1}^{2} A_{k_{l}}^{\left(s_{j}\right)} E^{2-s_{j}}, \quad(j=1,2),
$$

that satisfy, as (24), the commutation relations

$$
E A_{k_{l}}^{\left(s_{j}\right)}=e^{i k_{l}} A_{k_{l}}^{\left(s_{j}\right)} E, \quad(j, l=1,2) .
$$

The energy $\varepsilon_{\left\{s_{1}, s_{2}\right\}}$ end momentum $P$ are related to the spectral parameters by (25) and (27), respectively, and from (49) and (50) we have the commutation relations, generalizing (26),

$$
A_{k_{l}}^{\left(s_{j}\right)} \Omega_{P}=e^{i P\left(1-s_{j}\right)} \Omega_{P} A_{k_{l}}^{\left(s_{j}\right)}, \quad(j, l=1, \ldots, n),
$$

with $n=2$.

If the two particles are at the closest distance $x_{2}=$ $x_{1}+s_{1}$ ("matching" condition) the expression (28) should be replaced by

$$
\begin{aligned}
& \varepsilon_{\left\{s_{1}, s_{2}\right\}} \operatorname{Tr}\left(E^{x_{1}-1} A^{\left(s_{1}\right)} E^{s_{1}-1} A^{\left(s_{2}\right)} E^{L-x_{1}-s_{1}} \Omega_{P}\right)=-\epsilon_{+} \operatorname{Tr}\left(E^{x_{1}-2} A^{\left(s_{1}\right)} E^{s_{1}} A^{\left(s_{2}\right)} E^{L-x_{1}-s_{1}} \Omega_{P}\right) \\
& -\epsilon_{-} \operatorname{Tr}\left(E^{x_{1}-1} A^{\left(s_{1}\right)} E^{s_{1}} A^{\left(s_{2}\right)} E^{L-x_{1}-s_{1}-1} \Omega_{P}\right)+\operatorname{Tr}\left(E^{x_{1}-1} A^{\left(s_{1}\right)} E^{s_{1}-1} A^{\left(s_{2}\right)} E^{L-x_{1}-s_{1}} \Omega_{P}\right) .
\end{aligned}
$$

Inserting the definition (50), the expression (25) for $\varepsilon_{\left\{s_{1}, s_{2}\right\}}$ and using the algebraic relations (51) and (52), we obtain the commutation relations for the matrices $\left\{A_{k_{j}}^{\left(s_{l}\right)}\right\}$,

$$
A_{k_{j}}^{\left(s_{l}\right)} A_{k_{m}}^{\left(s_{r}\right)}=S\left(k_{j}, k_{m}\right) A_{k_{m}}^{\left(s_{l}\right)} A_{k_{j}}^{\left(s_{r}\right)}, \quad(j \neq m ; l, r=1,2), \quad A_{k_{j}}^{\left(s_{1}\right)} A_{k_{j}}^{\left(s_{2}\right)}=0,
$$

where $S\left(k_{j}, k_{m}\right)$ is given by the same expression as (31). It is interesting to notice that the structure constants $S\left(k_{j}, k_{m}\right)$ of the algebra in (53) are independent of the superscript of the matrices $A_{k_{j}}^{\left(s_{l}\right)}$, and consequently the algebra among the $\left\{A_{k_{j}}^{\left(s_{l}\right)}\right\}$ is the same as that of (30) with respect to the interchange of spectral parameters. However, the superscript of these matrices cannot be neglected since in the com- mutations relations (53) they are not interchanged and also their commutation with the $\Omega_{P}$ matrix is size dependent (see (52)).

The spectral parameters $k_{1}$ and $k_{2}$ are fixed by the cyclic property of the trace, and we have, mutatis mutandis, a similar expression as (32). Using the algebraic relations (51), (52) and (53) we obtain 


$$
\begin{aligned}
& \operatorname{Tr}\left(A_{k_{l}}^{\left(s_{1}\right)} A_{k_{j}}^{\left(s_{2}\right)} E^{L-s_{1}-s_{2}+2} \Omega_{P}\right)= \\
& e^{-i k_{j} L} e^{i k_{j}\left(s_{1}+s_{2}-2\right)} e^{-i P\left(s_{2}-1\right)} S\left(k_{j}, k_{l}\right) \operatorname{Tr}\left(A_{k_{l}}^{\left(s_{2}\right)} A_{k_{j}}^{\left(s_{1}\right)} E^{L-s_{1}-s_{2}+2} \Omega_{P}\right) .
\end{aligned}
$$

However, differently from (32), the traces on the left and on the right-hand side of the equation are not the same if $s_{1} \neq s_{2}$. If we repeat once more the commutations that lead to (54), we obtain the same trace in both sides and, consequently,

$$
\left[e^{-i k_{j} L} e^{i k_{j}\left(s_{1}+s_{2}-2\right)} S\left(k_{j}, k_{l}\right)\right]^{2} e^{-i P\left(s_{1}+s_{2}-1\right)}=1 .
$$

Since $P=k_{1}+k_{2}$, this last expression is equivalent to

$$
e^{i k_{j} L}=e^{i \frac{2 \pi}{2} m}\left(\frac{e^{i k_{j}}}{e^{i k_{l}}}\right)^{\bar{s}-1} S\left(k_{j}, k_{l}\right), \quad m=0,1 ; \quad j \neq l=1,2 ; \quad s_{1} \neq s_{2},
$$

and

$$
\bar{s}=\frac{s_{1}+s_{2}}{2}
$$

is the average size of the two molecules. The expression (56) generalizes the expression (33) obtained for particles of equal sizes. We note however that since $m=0,1$ in (56) we have two times more solutions than the corresponding one (33) for particles of equal sizes. This indeed should be the case since particles of distinct sizes are distinguishable, and consequently the number of eigenfunctions is doubled as compared with the indistinguishable case $\left(s_{1}=s_{2}\right)$.

\section{General n.}

In this case we have a general distribution of particles with sizes $\left\{s_{1}, s_{2}, \ldots, s_{n}\right\}$ and the corresponding eigenfunctions are given by (46) and (48). The eigenvalue equation when applied to the components $\left|x_{1}, \ldots, x_{n}\right\rangle$, where all the particles are not at the closest distance, gives an equation similar to (34), mutatis mutandis, whose solution is given by the generalization of (50), (52) and (51),

$$
A^{\left(s_{j}\right)}=\sum_{l=1}^{n} A_{k_{l}}^{\left(s_{j}\right)} E^{2-s_{j}}, \quad A_{k_{l}}^{\left(s_{j}\right)} \Omega_{P}=e^{i P\left(1-s_{j}\right)} \Omega_{P} A_{k_{l}}^{\left(s_{j}\right)}, \quad E A_{k_{l}}^{\left(s_{j}\right)}=e^{i k_{l}} A_{k_{l}}^{\left(s_{j}\right)} E, \quad(j=1,2),
$$

producing the energy and momentum given by

$$
\varepsilon_{n}=\sum_{j=1}^{n}\left(\epsilon_{+} e^{-i k_{j}}+\epsilon_{-} e^{i k_{j}}-1\right), \quad P=\sum_{j=1}^{n} k_{j},
$$

respectively. The eigenvalue equation applied to the components where a pair of particles $\left(x_{i}, x_{i+1}\right)$ are located at the closest distance, $x_{i+1}=x_{i}+s_{i}$ will give a generalization of (53),

$$
A_{k_{j}}^{\left(s_{t}\right)} A_{k_{l}}^{\left(s_{u}\right)}=S\left(k_{j}, k_{l}\right) A_{k_{l}}^{\left(s_{t}\right)} A_{k_{j}}^{\left(s_{u}\right)}, \quad(j \neq l), \quad A_{k_{j}}^{\left(s_{t}\right)} A_{k_{j}}^{\left(s_{u}\right)}=0, \quad(j, l, t, u=1, \ldots, n) .
$$

The cyclic property of the trace in (48) will give, by using (58) and (60), a generalization of (54), namely, for each $k_{j}$,

$$
\begin{aligned}
& \operatorname{Tr}\left(A_{k_{1}}^{\left(s_{1}\right)} A_{k_{2}}^{\left(s_{2}\right)} \cdots A_{k_{j-1}}^{\left(s_{j-1}\right)} A_{k_{j}}^{\left(s_{j}\right)} \cdots A_{k_{n}}^{\left(s_{n}\right)} E^{L-\sum_{i=1}^{n}\left(s_{i}-1\right)} \Omega_{P}\right)=e^{-i k_{j} L} e^{i k_{j} \sum_{i=1}^{n}\left(s_{i}-1\right)} e^{-i P\left(s_{j}-1\right)} \times \\
& \left(\prod_{l=1}^{n} S\left(k_{j}, k_{l}\right)\right) \operatorname{Tr}\left(A_{k_{1}}^{\left(s_{n}\right)} A_{k_{2}}^{\left(s_{1}\right)} \cdots A_{k_{j}}^{\left(s_{j-1}\right)} A_{k_{j+1}}^{\left(s_{j}\right)} \cdots A_{k_{n}}^{\left(s_{n-1}\right)} E^{L-\sum_{i=1}^{n}\left(s_{i}-1\right)} \Omega_{P}\right) .
\end{aligned}
$$

Similarly as happened in (54) the traces in both sides of the last equation are not the same because $\left\{s_{1}, s_{2}, \ldots, s_{n}\right\} \neq$ $\left\{s_{n}, s_{1}, \ldots, s_{n-1}\right\}$. But we can do the above commutations $r$ times until we reach the same distribution of sizes, where $r$ is the minimum number of cyclic rotations of $\left\{s_{1}, s_{2}, \ldots, s_{n}\right\}$ where the configuration repeats the initial one. In this case we obtain

$$
\left[e^{-i k_{j} L} e^{i k_{j} \sum_{i=1}^{n}\left(s_{i}-1\right)} \prod_{l=1}^{n} S\left(k_{j}, k_{l}\right)\right]^{r} e^{-i P \frac{r}{n} \sum_{i=1}^{n}\left(s_{i}-1\right)}=1 .
$$

Since $P=\sum_{i=1}^{n} k_{j}$, we can rewrite this last expression as

$$
e^{i k_{j} L}=e^{i \frac{2 \pi}{r} m} \prod_{l=1}^{n} S\left(k_{j}, k_{l}\right)\left(\frac{e^{i k_{j}}}{e^{i k_{l}}}\right)^{\bar{s}-1}, \quad m=0,1, \ldots, r-1, \quad j, l=1, \ldots, n,
$$


where, as in (57),

$$
\bar{s}=\frac{1}{n} \sum_{i=1}^{n} s_{i}
$$

is the average size of the molecules in the distribution $\left\{s_{1}, s_{2}, \ldots, s_{n}\right\}$. This equation gives us a number of solutions of order $r$ times larger than the corresponding number in the case where all the particles have the same size.

The equation (63) that sets the spectral parameters of the matrices coincides with the Bethe - ansatz equations derived in [15]. Similarly as we did in (40) - (45), we can show that indeed the eigenfunctions we obtained by using our matrix product ansatz coincide with the ones derived in the framework of the coordinate Bethe ansatz.

\section{The asymmetric diffusion model with $N$ classes of particles with hi- erarchical order}

The extension of the simple exclusion problem to the case where we have $N$ distinct classes of particles ( $c=$ $1,2, \ldots, N)$ diffusing asymmetrically is not exactly integrable in general. However the integrability of the problem can be preserved if the diffusive transitions of the several species happen in an hierarchical order. This problem was considered originally for the case $N=2$ as a model to describe shocks [25]-[27] in nonequilibrium. The stationary properties of the $N=2$ [31] and $N=3$ [43] models can also be studied through a matrix product ansatz. In [19] a generalization of this problem was considered in which the particles in each of the $N$ classes $(c=1,2, \ldots, N)$ may have distinct sizes $\left(s_{1}, \ldots, s_{N}\right)$, respectively. The solution of this generalized problem was obtained through the coordinate Bethe ansatz [19]. In the next section, we are going to show that the solution of this problem, similarly as we did in the last section, can also be obtained through an appropriate matrix product ansatz.

In this generalized problem we consider the particles in each class $c$ as composed by $s_{c}$ monomers, thus occupying $s_{c}$ sites on the lattice $(c=1,2, \ldots)$. We consider as the position of the molecule the coordinate of its leftmost monomer. The excluded volume of a particle in class $c$ is given by its size $s_{c}(c=1,2, \ldots, N)$ in units of lattice spacing. The configurations of the molecules in the lattice are described by defining at each lattice site $i$ a variable $\beta_{i}$ $(i=1,2, \ldots, L)$, taking the values $\beta_{i}=0,1, \ldots, N$. The values $\beta_{i}=1, \ldots, N$ represent sites occupied by molecules of class $c=1,2, \ldots, N$, respectively. The sites attached with the value $\beta=0$ are the vacant sites or those excluded due to the size of the particles. As an example, $\{\beta\}=$ $\{1,0,2,0,2,0\}$ may represent the configuration where in a $L=6$ sites we have a particle of class 1 and size $s_{1}=2$ at the site 1 and two particles of class 2 and size $s_{2}=1$ located at the sites 3 and 5 . The allowed configurations are given, in general, by the set $\left\{\beta_{i}\right\}(i=1,2, \ldots, L)$, where for each pair $\left(\beta_{1}, \beta_{j}\right) \neq 0$ with $j>i$ we have $j-i>s_{\beta_{i}}$. The allowed stochastic processes in the problem are just given by the exchange of particles or the asymmetric diffusion if the constraint due to the size of particles is satisfied. The possible motions of a given molecule are diffusion to the right,

$$
\beta_{i} \emptyset_{i+1} \rightarrow \emptyset_{i} \beta_{i+1} \quad\left(\text { rate } \Gamma_{R}\right)
$$

diffusion to the left,

$$
\emptyset_{i} \beta_{i+1} \rightarrow \beta_{i} \emptyset_{i+1} \quad\left(\text { rate } \Gamma_{L}\right)
$$

and interchange of particles,

$$
\begin{array}{lllll}
\beta_{i} \beta_{i+s_{\beta}}^{\prime} & \rightarrow \beta_{i}^{\prime} \beta_{i+s_{\beta^{\prime}}} \quad\left(\beta<\beta^{\prime}\right) & \left(\text { rate } \Gamma_{R}\right), \\
\beta_{i} \beta_{i+s_{\beta}}^{\prime} & \rightarrow \beta_{i}^{\prime} \beta_{i+s_{\beta^{\prime}}} \quad\left(\beta>\beta^{\prime}\right) & \left(\text { rate } \Gamma_{L}\right),(67
\end{array}
$$

with $\beta, \beta^{\prime}=1,2, \ldots, N$. As we see from (67), particles of a class $c$ interchange positions with those in classes $c^{\prime}>c$ with the same rate as they, interchange, positions with the vacant sites (diffusion). However, the net effect of these motions is distinct from the diffusion processes, since by interchanging positions, distinctly from the diffusion process, the particles move by $s_{c^{\prime}}$ lattice units, accelerating its diffusion if $s_{c^{\prime}}>1$. The identification of the master equation as a Schrödinger equation as in (5) will give us the Hamiltonian [19]

$$
\begin{aligned}
H= & D \mathcal{P} \sum_{j=1}^{L} H_{j} \mathcal{P} \\
H_{j}= & -\left\{\sum_{\alpha=1}^{N}\left[\epsilon_{+}\left(1-E_{j}^{\alpha, 0} E_{j+1}^{0, \alpha}\right) \mathcal{P} E_{j}^{0, \alpha} E_{j+1}^{\alpha, 0}+\epsilon_{-}\left(1-E_{j}^{0, \alpha} E_{j+1}^{\alpha, 0}\right) \mathcal{P} E_{j}^{\alpha, 0} E_{j+1}^{0, \alpha}\right]\right. \\
& \left.+\sum_{\alpha=1}^{N} \sum_{\beta=1}^{N} \epsilon_{\alpha, \beta}\left(E_{j}^{\beta, \alpha} E_{j+s_{\beta}}^{\alpha, 0} E_{j+s_{\alpha}}^{0, \beta}-E_{j}^{\alpha, \alpha} E_{j+s_{\beta}}^{0,0} E_{j+s_{\alpha}}^{\beta, \beta}\right)\right\},
\end{aligned}
$$

with

$$
\begin{gathered}
D=\Gamma_{R}+\Gamma_{L}, \quad \epsilon_{+}=\frac{\Gamma_{R}}{\Gamma_{R}+\Gamma_{L}}, \quad \epsilon_{-}=\frac{\Gamma_{L}}{\Gamma_{R}+\Gamma_{L}} \quad\left(\epsilon_{+}+\epsilon_{-}=1\right), \\
\epsilon_{\alpha \beta}= \begin{cases}\epsilon_{+}, & \alpha<\beta, \\
0, & \alpha=\beta, \\
\epsilon_{-}, & \alpha>\beta,\end{cases}
\end{gathered}
$$


and periodic boundary conditions. The matrices $E^{\alpha, \beta}$ are $(N+1) \times(N+1)$-dimensional matrices with a single nonzero element $\left(E^{\alpha, \beta}\right)_{i j}=\delta_{\alpha i} \delta_{\beta j}(\alpha, \beta, i, j=0, \ldots, N)$. The projector $\mathcal{P}$ in (68) projects out the configurations associated with the vectors $|\{\beta\}\rangle$ representing molecules at forbidden positions due to their finite size. Mathematically, this condition means that for all $i, j$ with $\beta_{i}, \beta_{j} \neq 0$ we should have $|i-j| \geq s_{\beta_{i}}(j>i)$. The constant $D$ in (68) fixes the time scale in the problem, and we chose $D=1$. The Hamiltonian (68) corresponding to the particular case where all the molecules have unit size is related to the spin$N / 2 \mathrm{SU}(N+1)$ anisotropic Sutherland chain [28, 44] or $\mathrm{SU}(N+1)$ Perk-Schultz model [29] with twisted boundary conditions [3].
At the end of the next section, we are going to present the solution of an even further generalized model whose solution was not derived in [19]. The solution of this model is quite complicated through the standard coordinate Bethe ansatz. As we shall see, however, its derivation is not difficult through our matrix product ansatz. In this generalization, instead of having all the particles in a given class $c$ with fixed size $s_{c}$, each individual particle may have an arbitrary size. In this case the configurations on the lattice are given by $\{\vec{\beta}\}=\left\{\vec{\beta}_{1}, \vec{\beta}_{2}, \ldots, \vec{\beta}_{L}\right\}$ where $\vec{\beta}_{i}=(c, s)$ means that the lattice site $i(i=1,2, \ldots, L)$ is occupied by a particle of class $c(c=1,2, \ldots, N)$ having size $s(s=1,2, \ldots)$. The Hamiltonian related to this stochastic problem is given by a generalization of (68), namely

$$
\begin{aligned}
H= & D \mathcal{P} \sum_{j=1}^{L} H_{j} \mathcal{P}, \\
H_{j}= & -\left\{\sum_{\vec{\beta}}\left[\epsilon_{+}\left(1-E_{j}^{\vec{\beta}, \overrightarrow{0}} E_{j+1}^{\overrightarrow{0}, \vec{\beta}}\right) \mathcal{P} E_{j}^{\overrightarrow{0}, \vec{\beta}} E_{j+1}^{\vec{\beta}, \overrightarrow{0}}+\epsilon_{-}\left(1-E_{j}^{\overrightarrow{0}, \vec{\beta}} E_{j+1}^{\vec{\beta}, \overrightarrow{0}}\right) \mathcal{P} E_{j}^{\vec{\beta}, \overrightarrow{0}} E_{j+1}^{0, \vec{\beta}}\right]\right. \\
& \left.+\sum_{\vec{\beta}=(c, s)} \sum_{\vec{\beta}^{\prime}=\left(c^{\prime}, s^{\prime}\right)} \epsilon_{c c^{\prime}}\left(E_{j}^{\vec{\beta}^{\prime}, \vec{\beta}} E_{j+s^{\prime}}^{\vec{\beta}, \overrightarrow{0}} E_{j+s}^{\overrightarrow{0}, \vec{\beta}^{\prime}}-E_{j}^{\vec{\beta}, \vec{\beta}} E_{j+s^{\prime}}^{0, \overrightarrow{0}} E_{j+s}^{\vec{\beta}^{\prime}, \vec{\beta}^{\prime}}\right)\right\},
\end{aligned}
$$

with $\epsilon_{+}, \epsilon_{-}$and $\epsilon_{c c^{\prime}}\left(c, c^{\prime}=1,2, \ldots, N\right)$ given as in (69) and (70).

\section{A matrix product ansatz for the generalized diffusion problem with $N$ classes of particles with hierar- chical order}

The exact solution of the asymmetric diffusion problem with $N$ classes of particles, whose related Hamiltonian is given by (69) was obtained in [19] through the coordinate Bethe ansatz. In this section we are going to reobtain this solution by an appropriate matrix product ansatz. Moreover our solution enables the extension to the more general problem discussed in the last section and whose Hamiltonian was introduced in (71).

Let us initially consider the simple case where all the particles in a given class $(c=1, \ldots, N)$ have a fixed size $\left(s_{c}=1,2, \ldots\right)$. Due to the conservation of particles in the diffusion and interchange processes, the total number of particles in each class is conserved separately and we can split the associated Hilbert space into block disjoint eigensectors labeled by the numbers $n_{1}, n_{2}, \ldots, n_{N}\left(n_{i}=0,1, \ldots\right)$ of particles on the classes $i(i=1,2, \ldots, N)$. We want to obtain the eigenfunctions $\left|n_{1}, \ldots, n_{N}\right\rangle$ of the eigenvalue equation

$$
H\left|n_{1}, \ldots, n_{N}\right\rangle=\varepsilon_{n}\left|n_{1}, \ldots, n_{N}\right\rangle,
$$

where

$$
\left|n_{1}, \ldots, n_{N}\right\rangle=\sum_{\{c\}} \sum_{\{x\}}^{*} f\left(x_{1}, c_{1} ; \ldots ; x_{n}, c_{n}\right)\left|x_{1}, c_{1} ; \ldots ; x_{n}, c_{n}\right\rangle
$$

The ket $\left|x_{1}, c_{1} ; \ldots ; x_{n}, c_{n}\right\rangle$ means the configuration where particles of class $c_{i}\left(c_{i}=1, \ldots, N\right)$ are located at position $x_{i}\left(x_{i}=1, \ldots, L\right)$ and the total number of particles is $n=n_{1}+\cdots+n_{N}$. The summation $\{c\}=$ $\left\{c_{1}, \ldots, c_{n}\right\}$ extends over all the permutations of $n$ integer numbers $\{1,2, \ldots, N\}$ in which $n_{i}$ terms have the value $i$ $(i=1, \ldots, N)$, while the summation $\{x\}=\left\{x_{1}, \ldots, x_{n}\right\}$ runs, for each permutation $\{c\}$, over the set of the nonde- 
creasing integers satisfying

$$
\begin{aligned}
x_{i+1} & \geq x_{i}+s_{c_{i}}, \quad i=1, \ldots, n-1, \\
s_{c_{1}} & \leq x_{n}-x_{1} \leq L-s_{c_{n}} .
\end{aligned}
$$

The matrix product ansatz we propose asserts that an arbitrary eigenfunction $\left|n_{1}, \ldots, n_{N}\right\rangle$ with momentum $P$, will have the amplitudes in (73) given in terms of traces of the matrix product

$$
f\left(x_{1}, c_{1} ; \ldots ; x_{n}, c_{n}\right)=\operatorname{Tr}\left[E^{x_{1}-1} Y^{\left(c_{1}\right)} E^{x_{2}-x_{1}-1} Y^{\left(c_{2}\right)} \cdots E^{x_{n}-x_{n-1}-1} Y^{\left(c_{n}\right)} E^{L-x_{n}} \Omega_{P}\right] .
$$

The matrices $Y^{(c)}(c=1, \ldots, N), E$ and $\Omega_{P}$ will obey algebraic relations that ensure the validity of the eigenvalue equation (72). The momentum $P$ of the state, analogously as in Sec. 3 , is fixed by requiring the relation

$$
E \Omega_{P}=e^{-i P} \Omega_{P} E, \quad Y^{(c)} \Omega_{P}=e^{-i P} \Omega_{P} Y^{(c)}, \quad c=1, \ldots, N .
$$

Let us consider the simplest cases of $n=1$ and $n=2$ particles before considering the case where $n$ is general.

$\mathbf{n}=\mathbf{1}$

In this case the problem is the same as that of section 3 and we obtain the energies given by (18).

$\mathbf{n}=\mathbf{2}$.

For two particles of classes $c_{1}$ and $c_{2}\left(c_{1}, c_{2}=1, \ldots, N\right)$ on the lattice we have two distinct types of relations depending if the amplitudes are related or not to the configurations where two particles are at the closest distance $x_{2}=x_{1}+s_{c_{1}}$. The eigenvalue equation when applied to the components where the particles of class $c_{1}$ and $c_{2}$ are at positions $\left(x_{2}, x_{1}\right)$, such that $x_{2}>x_{1}+s_{c_{1}}$, gives the relation

$$
\begin{aligned}
& \varepsilon_{2} \operatorname{Tr}\left[E^{x_{1}-1} Y^{\left(c_{1}\right)} E^{x_{2}-x_{1}-1} Y^{\left(c_{2}\right)} E^{L-x_{2}} \Omega_{P}\right]=-\epsilon_{+} \operatorname{Tr}\left[E^{x_{1}-2} Y^{\left(c_{1}\right)} E^{x_{2}-x_{1}} Y^{\left(c_{2}\right)} E^{L-x_{2}} \Omega_{P}\right] \\
& -\epsilon_{-} \operatorname{Tr}\left[E^{x_{1}} Y^{\left(c_{1}\right)} E^{x_{2}-x_{1}-2} Y^{\left(c_{2}\right)} E^{L-x_{2}} \Omega_{P}\right]-\epsilon_{+} \operatorname{Tr}\left[E^{x_{1}-1} Y^{\left(c_{1}\right)} E^{x_{2}-x_{1}-2} Y^{\left(c_{2}\right)} E^{L-x_{2}+1} \Omega_{P}\right] \\
& -\epsilon_{-} \operatorname{Tr}\left[E^{x_{1}-1} Y^{\left(c_{1}\right)} E^{x_{2}-x_{1}} Y^{\left(c_{2}\right)} E^{L-x_{2}-1} \Omega_{P}\right]+2 \operatorname{Tr}\left[E^{x_{1}-1} Y^{\left(c_{1}\right)} E^{x_{2}-x_{1}-1} Y^{\left(c_{2}\right)} E^{L-x_{2}} \Omega_{P}\right] .
\end{aligned}
$$

A solution of this equation is obtained by identifying the matrices $Y^{(c)}$ as composed by two spectral parameter-dependent new matrices $Y_{k_{1}}^{(c)}$ and $Y_{k_{2}}^{(c)}$, i. e. ,

$$
Y^{(c)}=\sum_{i=1}^{2} Y_{k_{i}}^{(c)} E^{2-s_{c}},
$$

which, as in Eq. (24), satisfies the commutation relation

$$
E Y_{k_{j}}^{(c)}=e^{i k_{j}} Y_{k_{j}}^{(c)} E .
$$

In terms of the unknown spectral parameters $k_{j}(j=1,2)$, the energy and momentum are given by

$$
\varepsilon_{2}=\varepsilon\left(k_{1}\right)+\varepsilon\left(k_{2}\right), \quad P=k_{1}+k_{2},
$$

where $\varepsilon(k)=-\left(\epsilon_{+} e^{-i k}+\epsilon_{-} e^{i k}-1\right)$. As a consequence of (76) and (78), we also have

$$
Y_{k_{j}}^{(c)} \Omega_{P}=e^{i P\left(1-s_{c}\right)} \Omega_{P} Y_{k_{j}}^{(c)} \quad(j=1,2 ; c=1, \ldots, N) .
$$

The eigenvalue equation (72) when applied to the components of (73) where the two particles are at the closest distance, i. e., $x_{2}=x_{1}+s_{c_{1}}$, gives

$$
\begin{aligned}
& \varepsilon_{2} \operatorname{Tr}\left[E^{x_{1}-1} Y^{\left(c_{1}\right)} E^{s_{c_{1}}-1} Y^{\left(c_{2}\right)} E^{\left.L-x_{1}-s_{c_{1}} \Omega_{P}\right]}=-\epsilon_{+} \operatorname{Tr}\left[E^{x_{1}-2} Y^{\left(c_{1}\right)} E^{s_{c_{1}}} Y^{\left(c_{2}\right)} E^{\left.L-x_{1}-s_{c_{1}} \Omega_{P}\right]}\right.\right. \\
- & \epsilon_{-} \operatorname{Tr}\left[E^{x_{1}-1} Y^{\left(c_{1}\right)} E^{s_{c_{1}}} Y^{\left(c_{2}\right)} E^{L-x_{1}-s_{c_{1}}-1} \Omega_{P}\right]-\epsilon_{c_{2} c_{1}} \operatorname{Tr}\left[E^{x_{1}-1} Y^{\left(c_{2}\right)} E^{s_{c_{2}}-1} Y^{\left(c_{1}\right)} E^{\left.L-x_{1}-s_{c_{2}} \Omega_{P}\right]}\right. \\
& +2\left(1+\epsilon_{c_{1} c_{2}}\right) \operatorname{Tr}\left[E^{x_{1}-1} Y^{\left(c_{1}\right)} E^{s_{c_{1}}-1} Y^{\left(c_{2}\right)} E^{\left.L-x_{1}-s_{c_{1}} \Omega_{P}\right] .}\right.
\end{aligned}
$$

Substituting (78) and (80) in this last expression and using (79) we obtain

$$
\begin{aligned}
& \sum_{l, m}\left\{\left[-\left(\epsilon_{+} e^{-i\left(k_{l}+k_{m}\right)}+\epsilon_{-}\right)+e^{-i k_{l}}\left(1-\epsilon_{c_{1}, c_{2}}\right)\right] \operatorname{Tr}\left[E^{x_{1}} Y_{k_{l}}^{\left(c_{1}\right)} Y_{k_{m}}^{\left(c_{2}\right)} E^{L-x_{1}-s_{c_{1}}-s_{c_{2}}+2} \Omega_{P}\right]\right. \\
& \left.+\epsilon_{c_{2}, c_{1}} e^{-i k_{l}} \operatorname{Tr}\left[E^{x_{1}} Y_{k_{l}}^{\left(c_{2}\right)} Y_{k_{m}}^{\left(c_{1}\right)} E^{L-x_{1}-s_{c_{1}}-s_{c_{2}}+2} \Omega_{P}\right]\right\}=0 .
\end{aligned}
$$


This last equation is satisfied by imposing the following commutation relations among the operators $\left\{Y_{k}^{(c)}\right\}$,

$$
\sum_{l} \sum_{m}\left\{\left[\mathcal{D}_{l, m}+e^{i k_{m}}\left(1-\epsilon_{c_{1}, c_{2}}\right)\right] Y_{k_{l}}^{\left(c_{1}\right)} Y_{k_{m}}^{\left(c_{2}\right)}+\epsilon_{c_{2}, c_{1}} e^{i k_{m}} Y_{k_{l}}^{\left(c_{2}\right)} Y_{k_{m}}^{\left(c_{1}\right)}\right\}=0
$$

where

$$
\mathcal{D}_{l, m}=-\left(\epsilon_{+}+\epsilon_{-} e^{i\left(k_{l}+k_{m}\right)}\right) .
$$

It is interesting to consider separately the cases where the two particles belong to the same class $c_{1}=c_{2}$ from the case where $c_{1} \neq c_{2}$. If $c_{1}=c_{2}=c(c=1, \ldots, N)$, since $\epsilon_{c, c}=0$ and $\mathcal{D}_{l, m}+e^{i k_{m}} \neq \mathcal{D}_{m, l}+e^{i k_{l}}$ for $l \neq m$, we obtain

$$
Y_{k_{l}}^{(c)} Y_{k_{m}}^{(c)}=S_{c, c}^{c, c}\left(k_{l}, k_{m}\right) Y_{k_{m}}^{(c)} Y_{k_{l}}^{(c)} \quad(l \neq m), \quad\left(Y_{k_{l}}^{(c)}\right)^{2}=0,
$$

where

$$
S_{c, c}^{c, c}\left(k_{l}, k_{m}\right)=-\frac{\epsilon_{+}+\epsilon_{-} e^{i\left(k_{l}+k_{m}\right)}-e^{i k_{l}}}{\epsilon_{+}+\epsilon_{-} e^{i\left(k_{l}+k_{m}\right)}-e^{i k_{m}}}
$$

and $(l, m=1,2 ; c=1, \ldots, N)$. The relation (84) in the cases where $c_{1} \neq c_{2}$ gives the equations in matrix form,

$$
\sum_{l, m=1}^{2}\left[\begin{array}{cc}
\mathcal{D}_{l, m}+\epsilon_{c_{2}, c_{1}} e^{i k_{m}} & \epsilon_{c_{2}, c_{1}} e^{i k_{m}} \\
\epsilon_{c_{1}, c_{2}} e^{i k_{m}} & \mathcal{D}_{l, m}+\epsilon_{c_{1}, c_{2}} e^{i k_{m}}
\end{array}\right]\left[\begin{array}{c}
Y_{k_{l}}^{\left(c_{1}\right)} Y_{k_{m}}^{\left(c_{2}\right)} \\
Y_{k_{l}}^{\left(c_{2}\right)} Y_{k_{m}}^{\left(c_{1}\right)}
\end{array}\right]=0
$$

Similarly as in [19], the above equation can be rearranged straightforwardly by giving us the algebraic relations

$$
\begin{aligned}
Y_{k_{l}}^{\left(c_{1}\right)} Y_{k_{m}}^{\left(c_{2}\right)} & =\sum_{c_{1}^{\prime}, c_{2}^{\prime}=1}^{N} S_{c_{1}^{\prime}, c_{2}^{\prime}}^{c_{1}, c_{2}}\left(k_{l}, k_{m}\right) Y_{k_{m}}^{\left(c_{2}^{\prime}\right)} Y_{k_{l}}^{\left(c_{1}^{\prime}\right)}\left(k_{l} \neq k_{m}\right), \\
Y_{k_{l}}^{\left(c_{1}\right)} Y_{k_{l}}^{\left(c_{2}\right)} & =0,
\end{aligned}
$$

where $(l, m=1,2), c_{1}, c_{2}=1, \ldots, N$ and the "structure constants" of the algebra are the components of an $S$-matrix whose non-zero components are given by (87) and

$$
\begin{aligned}
& S_{c_{2}, c_{1}}^{c_{1}, c_{2}}\left(k_{1}, k_{2}\right)=\left[1-\epsilon_{c_{1}, c_{2}} \Phi\left(k_{1}, k_{2}\right)\right] S_{c_{1}, c_{1}}^{c_{1}, c_{1}}\left(k_{1}, k_{2}\right) \quad\left(c_{1}, c_{2}=1, \ldots, N\right), \\
& S_{c_{1}, c_{2}}^{c_{1}, c_{2}}\left(k_{1}, k_{2}\right)=\epsilon_{c_{2}, c_{1}} \Phi\left(k_{1}, k_{2}\right) S_{c_{1}, c_{1}}^{c_{1}, c_{1}}\left(k_{1}, k_{2}\right) \quad\left(c_{1}, c_{2}=1, \ldots, N ; \quad c_{1} \neq c_{2}\right),
\end{aligned}
$$

where

$$
\Phi\left(k_{1}, k_{2}\right)=\frac{e^{i k_{1}}-e^{i k_{2}}}{e^{i k_{1}}-\epsilon_{+}-\epsilon_{-} e^{\left(i k_{1}+k_{2}\right)}} .
$$

The complex parameters $\left(k_{1}, k_{2}\right)$, that are free up to now, are going to be fixed by the cyclic property of the trace in (75) and the algebraic relations (76), (78), (79) and (81).

Instead of solving for the spectral parameters for $n=2$, let us consider the case of general $n$.

\section{General n}

In this case the eigenvalue equation (72), when applied to the components of the eigenfunction corresponding to the configuration where all particles are at distances larger than the closest distance, gives a generalization of (77) that is promptly solved by identifying, as in (78), the matrices $Y^{(c)}$ as combinations of $n$ spectral parameter matrices,

$$
Y^{(c)}=\sum_{i=1}^{n} Y_{k_{i}}^{(c)} E^{2-s_{c}}
$$

satisfying the following algebraic relations with the matrices
$E$

$$
E Y_{k_{j}}^{(c)}=e^{i k_{j}} Y_{k_{j}}^{(c)} E(j=1, \ldots, n ; c=1,2, \ldots, N),
$$

and, from (76),

$$
\begin{gathered}
Y_{k_{j}}^{(c)} \Omega_{P}=e^{i P\left(1-s_{c}\right)} \Omega_{P} Y_{k_{j}}^{(c)} \\
(j=1, \ldots, n ; c=1,2, \ldots, N) .
\end{gathered}
$$

The energy and momentum in terms of the spectral parameter $\left\{k_{j}\right\}$ are given by the generalizations of (80), namely,

$$
\varepsilon_{n}=\sum_{j=1}^{n} \varepsilon\left(k_{j}\right), \quad P=\sum_{j=1}^{n} k_{j} .
$$

The components of the eigenfunctions corresponding to the configurations where a pair of particles of classes $c_{1}$ and $c_{2}$ are located at the closest positions $x_{i}$ and $x_{i+1}=x_{i}+s_{c_{1}}$ will give relations that reproduce (89) - (91).

Since in the general case we have the product of $n$ operators $\left\{Y_{k_{j}}^{(c)}\right\}$, the algebraic relations (76), (79), (81) and (89) should provide a unique relation among these products. For 
example the product $\cdots Y_{k_{1}}^{(\alpha)} Y_{k_{2}}^{(\beta)} Y_{k_{3}}^{(\gamma)} \cdots$ can be related to the product $\cdots Y_{k_{3}}^{(\gamma)} Y_{k_{2}}^{(\beta)} Y_{k_{1}}^{(\alpha)} \cdots$ by two distinct ways.
Either by performing the commutations $\alpha \beta \gamma \rightarrow \beta \alpha \gamma \rightarrow$ $\beta \gamma \alpha \rightarrow \gamma \beta \alpha$ or by $\alpha \beta \gamma \rightarrow \alpha \gamma \beta \rightarrow \gamma \alpha \beta \rightarrow \gamma \beta \alpha$. Consequently, we should have

$$
\begin{array}{ll}
\sum_{\gamma, \gamma^{\prime}, \gamma^{\prime \prime}=1}^{N} \quad & S_{\gamma, \gamma^{\prime}}^{\alpha, \alpha^{\prime}}\left(k_{1}, k_{2}\right) S_{\beta, \gamma^{\prime \prime}}^{\gamma, \alpha^{\prime \prime}}\left(k_{1}, k_{3}\right) S_{\beta^{\prime}, \beta^{\prime \prime}}^{\gamma^{\prime}, \gamma^{\prime \prime}}\left(k_{2}, k_{3}\right)= \\
& \sum_{\gamma, \gamma^{\prime}, \gamma^{\prime \prime}=1}^{N} S_{\gamma^{\prime}, \gamma^{\prime \prime}}^{\alpha^{\prime}, \alpha^{\prime \prime}}\left(k_{2}, k_{3}\right) S_{\gamma, \beta^{\prime \prime}}^{\alpha, \gamma^{\prime \prime}}\left(k_{1}, k_{3}\right) S_{\beta, \beta^{\prime}}^{\gamma, \gamma^{\prime}}\left(k_{1}, k_{2}\right),
\end{array}
$$

for $\alpha, \alpha^{\prime}, \alpha^{\prime \prime}, \beta, \beta^{\prime}, \beta^{\prime \prime}=1, \ldots, N$. This last relation is just the Yang-Baxter relations $[42,21]$ of the $S$-matrix defined in (87) and (90). Actually, the condition (96) is enough to ensure that any matrix product is uniquely related and it implies the associativity of the algebra of the operators $\left\{Y_{k_{j}}^{(c)}\right\}$. We can verify that the Yang-Baxter relation (96), with $S$ given by (87) and (90), is satisfied by an arbitrary number of distinct species of particles $N$. It is interesting to remark that in our solution, as compared to that presented in [19] through the coordinate Bethe ansatz, has the advantage that the derived $S$-matrix does not depend on the size of the particles and the associativity condition or Yang-Baxter relation (96) is easier to be verified since it is the same, independently of the particle sizes.

The spectral parameters $\left\{k_{j}\right\}$ are fixed by the cyclic property of the trace in (75). For each spectral parameter $k_{j}(j=1, \ldots, n)$, the commutations relation (76), (78), (79) and (81) applied $j$ times enable us to move the operator $Y_{k_{j}}^{\left(c_{j}\right)}$ to the left,

$$
\begin{aligned}
& \operatorname{Tr}\left[Y_{k_{1}}^{\left(c_{1}\right)} \cdots Y_{k_{j-1}}^{\left(c_{j-1}\right)} Y_{k_{j}}^{\left(c_{j}\right)} \cdots Y_{k_{n}}^{\left(c_{n}\right)} E^{L-\sum_{j=1}^{N} n_{j} s_{j}+n} \Omega_{P}\right]=e^{i k_{j}\left(L-\sum_{j=1}^{N} n_{j} s_{j}+n\right)} \\
& \quad \sum_{c_{j-1}^{\prime}, c_{j-1}^{\prime \prime}} \sum_{c_{j-2}^{\prime}, c_{j-2}^{\prime \prime}} \cdots \sum_{c_{1}^{\prime}, c_{1}^{\prime \prime}}\left\{e^{i P\left(s_{1}^{\prime \prime}-1\right)} S_{c_{1}^{\prime}, c_{1}^{\prime \prime}}^{c_{1}, c_{1}^{\prime \prime}}\left(k_{1}, k_{j}\right) \cdots S_{c_{j-2}^{\prime}, c_{j-2}^{\prime \prime}}^{c_{j-2}, c_{j-1}^{\prime \prime}}\left(k_{j-2}, k_{j}\right) S_{c_{j-1}^{\prime}, c_{j-1}^{\prime \prime}}^{c_{j-1}, c_{j}}\left(k_{j-1}, k_{j}\right)\right. \\
& \left.\operatorname{Tr}\left[Y_{k_{1}}^{\left(c_{1}^{\prime}\right)} \cdots Y_{k_{j-1}}^{\left(c_{j-1}^{\prime}\right)} Y_{k_{j+1}}^{\left(c_{j+1}\right)} \cdots Y_{k_{n}}^{\left(c_{n}\right)} Y_{k_{j}}^{\left(c_{1}^{\prime \prime}\right)} E^{L-\sum_{j=1}^{N} n_{j} s_{j}+n} \Omega_{P}\right]\right\} .
\end{aligned}
$$

Moving the operator $Y_{k_{j}}^{\left(c_{1}^{\prime \prime}\right)}$ for additional $n-j$ times to the left and using the identity

$$
\sum_{c_{j}^{\prime \prime}, c_{j+1}^{\prime \prime}=1}^{N} S_{c_{j}^{\prime}, c_{j}^{\prime \prime}}^{c_{j}, c_{j+1}^{\prime \prime}}\left(k_{j}, k_{j}\right)=-1
$$

we can write

$$
\begin{aligned}
& \operatorname{Tr}\left[Y_{k_{1}}^{\left(c_{1}\right)} \cdots Y_{k_{n}}^{\left(c_{n}\right)} E^{L-\sum_{j=1}^{N} n_{j} s_{j}+n} \Omega_{P}\right]=e^{i k_{j}\left(L-\sum_{j=1}^{N} n_{j} s_{j}+n\right)} \\
& \sum_{c_{1}^{\prime}, \ldots, c_{n}^{\prime}}<c_{1}, \ldots, c_{n}|\mathcal{T}| c_{1}^{\prime}, \ldots, c_{n}^{\prime}>\operatorname{Tr}\left[Y_{k_{1}}^{\left(c_{1}^{\prime}\right)} \cdots Y_{k_{n}}^{\left(c_{n}^{\prime}\right)} E^{L-\sum_{j=1}^{N} n_{j} s_{j}+n} \Omega_{P}\right]
\end{aligned}
$$

where

$$
<\{c\}|\mathcal{T}|\left\{c^{\prime}\right\}>=\sum_{c_{1}^{\prime \prime}, \ldots, c_{n}^{\prime \prime}}\left\{S_{c_{1}^{\prime}, c_{1}^{\prime \prime}}^{c_{1}, c^{\prime \prime}}\left(k_{1}, k_{j}\right) \cdots S_{c_{j}^{\prime}, c_{j}^{\prime \prime}}^{c_{j} c_{j+1}^{\prime \prime}}\left(k_{j}, k_{j}\right) \cdots S_{c_{n}^{\prime}, c_{n}^{\prime \prime}}^{c_{n}, c_{n}^{\prime \prime}}\left(k_{n}, k_{j}\right) e^{i P\left(s_{1}^{\prime \prime}-1\right)}\right\}
$$


We identify in (100) $\mathcal{T}\left(k_{j} ;\left\{k_{l}\right\}\right)$ as the $N^{n} \times N^{n}$ dimensional transfer matrix of an inhomogeneous vertex model (inhomogeneities $\left\{k_{l}\right\}$ ) with Boltzmann weights given by (87) and (90). The model is defined on a cylinder of perimeter $n$ with a seam along its axis producing the twisted boundary condition

$$
S_{c_{n}^{\prime}, c_{n}^{\prime \prime}}^{c_{n}, c_{n}^{\prime \prime}}\left(k_{n}, k\right)=S_{c_{n}^{\prime}, c_{n}^{\prime \prime}}^{c_{n}, c_{n}^{\prime \prime}}\left(k_{n}, k\right) e^{i P\left(s_{1}^{\prime \prime}-1\right)}
$$

where as always $P$ is the momentum of the eigenstate. The relation (99) gives the conditions for the spectral parameters,

$$
e^{-i k_{j}\left(L+n-\sum_{i=1}^{N} n_{i} s_{i}\right)}=\Lambda\left(k_{j},\left\{k_{l}\right\}\right), \quad j=1, \ldots, n,
$$

where $\Lambda\left(k_{j},\left\{k_{l}\right\}\right)$ are the eigenvalues of the transfer matrix (100). The problem of fixing the spectral parameters $\left\{k_{j}\right\}$ reduces to the evaluation of the eigenvalues of the transfer matrix (100). This can be done straightforwardly through the coordinate Bethe ansatz as in [19]. Extracting these eigenvalues from [19] we obtain equations that coincide with the Bethe ansatz equations for this general model (see Eqs. (71)-(73) in [19]).

Let us consider the more general Hamiltonian given in (71), where now each molecule has an arbitrary size, independently of the sizes of the other molecules belonging to its class. The solution of this problem was not derived through the coordinate Bethe ansatz since it is not simple in that formulation. The Hamiltonian (71) is composed by block disjoint eigenvectors labeled by $\left\{c_{1}, s_{1} ; \ldots ; c_{n}, s_{n}\right\}$ $\left(c_{j}=1, \ldots, N ; s_{j}=1,2, \ldots ; j=1, \ldots, n\right)$ that specifies the classes and sizes of each individual particle. An arbitrary eigenfunction of (71) is given by a generalization of (73),

$$
\left|c_{1}, s_{1} ; \ldots ; c_{n}, s_{n}\right\rangle=\sum_{\{c, s\}} \sum_{\{x\}^{*}} f\left(x_{1}, c_{1}, s_{1} ; \ldots ; x_{n}, c_{n}, s_{n}\right)\left|x_{1}, c_{1}, s_{1} ; \ldots ; x_{n}, c_{n}, s_{n}\right\rangle
$$

where $\left|x_{1}, c_{1}, s_{1} ; \ldots ; x_{n}, c_{n}, s_{n}\right\rangle$ denotes the configuration where the particle located at $x_{i}(i=1, \ldots, L)$ belongs to the class $c_{i}\left(c_{i}=1, \ldots, N\right)$ and has size $s_{i}\left(s_{i}=1,2, \ldots\right)$. The summations $\{c, s\}=\left\{c_{p_{1}}, s_{p_{1}} ; \ldots ; c_{p_{n}}, s_{p_{n}}\right\}$ extend over all the permutations of particles. The summation $\{x\}^{*}=$ $\left\{x_{1}, \ldots, x_{n}\right\}^{*}$ runs, for each permutation $\{c, s\}$, over the set of non-decreasing integers satisfying

$$
\begin{aligned}
x_{i_{1}} & \geq x_{i}+s_{i}, \quad i=1, \ldots, n-1, \\
s_{1} & \leq x_{n}-x_{1} \leq L-s_{n} .
\end{aligned}
$$

Our matrix product ansatz asserts that the eigenfunctions with a given momentum $P$ have amplitudes

$$
\operatorname{Tr}\left[E^{x_{1}-1} Y^{\left(c_{1}, s_{1}\right)} E^{x_{2}-x_{1}-1} Y^{\left(c_{2}, s_{2}\right)} \cdots E^{x_{n}-x_{n-1}-1} Y^{\left(c_{n}, s_{n}\right)} E^{L-x_{n}} \Omega_{P}\right]
$$

where the matrices $E$ are associated with the empty sites, and $Y^{\left(c_{j}, s_{j}\right)}$ with the sites occupied by particles of class $c_{j}$ and having a size $s_{j}(j=1, \ldots, n)$. The matrices $\Omega_{P}$, as before, fix the momentum $P$ of the eigenstates,

$$
E \Omega_{P}=e^{-i P} \Omega_{P} E, \quad Y^{(c, s)} \Omega_{P}=e^{-i P} \Omega_{P} Y^{(c, s)} .
$$

The solution of this general problem follows, mutatis mutandis, the derivation we did in (77)-(102). The energy and momentum of the eigenstate are given by (95), where the spectral parameters are introduced by the generalization of (92),

$$
Y^{(c, s)}=\sum_{i=1}^{n} Y_{k_{i}}^{(c, s)} E^{2-s}
$$

where the spectral parameter matrices $Y_{k_{j}}^{(c, s)}$ satisfy the algebra

$$
E Y_{k_{j}}^{(c, s)}=e^{k_{j}} Y_{k_{j}}^{(c, s)} E \quad(j=1, \ldots, n ; c=1,2, \ldots, N ; s=1,2, \ldots),
$$

and, from (105),

$$
Y_{k_{j}}^{(c, s)} \Omega_{P}=e^{i P(1-s)} \Omega_{P} Y_{k_{j}}^{(c, s)} \quad(j=1, \ldots, n ; c=1,2, \ldots, N ; s=1,2, \ldots) .
$$

The algebraic relations among the $\left\{Y_{k_{j}}^{(c, s)}\right\}$ are given by the generalization of (89),

$$
\begin{aligned}
Y_{k_{l}}^{\left(c_{1}, s_{1}\right)} Y_{k_{m}}^{\left(c_{2}, s_{2}\right)} & =\sum_{c_{1}^{\prime}, c_{2}^{\prime}=1}^{N} S_{c_{1}^{\prime}, c_{2}^{\prime}}^{c_{1}, c_{2}}\left(k_{l}, k_{m}\right) Y_{k_{m}}^{\left(c_{2}^{\prime}, s_{1}\right)} Y_{k_{l}}^{\left(c_{1}^{\prime}, s_{2}\right)}\left(k_{l} \neq k_{m}\right), \\
Y_{k_{l}}^{\left(c_{1}, s_{1}\right)} Y_{k_{l}}^{\left(c_{2}, s_{2}\right)} & =0
\end{aligned}
$$


where $(l, m=1, \ldots, n), c_{1}, c_{2}=1, \ldots, N$ and $S_{c_{1}^{\prime}, c_{2}^{\prime}}^{c_{1}, c_{2}}\left(k_{l}, k_{m}\right)$ are the same $S$-matrix defined in (87), (90) and (91). It is interesting to observe that the condition of existence of a single relation among the words $\cdots Y_{k_{1}}^{\left(\alpha, s_{1}\right)} Y_{k_{2}}^{\left(\beta, s_{2}\right)} Y_{k_{3}}^{\left(\gamma, s_{3}\right)} \cdots$ and $\cdots Y_{k_{3}}^{\left(\gamma, s_{1}\right)} Y_{k_{2}}^{\left(\beta, s_{2}\right)} Y_{k_{3}}^{\left(\alpha, s_{3}\right)} \ldots$ reproduces the Yang-Baxter relation (96), as before.

The spectral parameters $\left\{k_{j}\right\}$, as before, are fixed by the cyclic property of the trace and the algebraic relations (105)-(109). Using these relations we can move the operator $Y_{k_{j}}^{\left(c_{j}, s_{j}\right)}$ to the left as in (97)-(100),

$$
\begin{aligned}
& \operatorname{Tr}\left[Y_{k_{1}}^{\left(c_{1}, s_{1}\right)} Y_{k_{2}}^{\left(c_{2}, s_{2}\right)} \cdots Y_{k_{n}}^{\left(c_{n}, s_{n}\right)} E^{L-\sum_{j=1}^{N}\left(s_{j}-1\right)} \Omega_{P}\right]=e^{i k_{j}\left[L-\sum_{j=1}^{N}\left(s_{j}-1\right)\right]} e^{i P\left(s_{1}-1\right)} \\
& \sum_{c_{1}^{\prime}, \ldots, c_{n}^{\prime}}<c_{1}, \ldots, c_{n}|\tilde{\mathcal{T}}| c_{1}^{\prime}, \ldots, c_{n}^{\prime}>\operatorname{Tr}\left[Y_{k_{1}}^{\left(c_{1}^{\prime}, s_{2}\right)} Y_{k_{2}}^{\left(c_{2}^{\prime}, s_{3}\right)} \cdots Y_{k_{n}}^{\left(c_{n}^{\prime}, s_{1}\right)} E^{L-\sum_{j=1}^{N}\left(s_{j}-1\right)} \Omega_{P}\right],
\end{aligned}
$$

where now

$$
<\{c\}|\tilde{\mathcal{T}}|\left\{c^{\prime}\right\}>=\sum_{c_{1}^{\prime \prime}, \cdots, c_{n}^{\prime \prime}}\left\{S_{c_{1}^{\prime}, c_{1}^{\prime \prime}}^{c_{1}, c_{2}^{\prime \prime}}\left(k_{1}, k_{j}\right) \cdots S_{c_{n}^{\prime}, c_{n}^{\prime \prime}}^{c_{n}, c_{1}^{\prime \prime}}\left(k_{n}, k_{j}\right)\right\}
$$

is different from (100), since it corresponds to a transfer matrix of a vertex model in a cylinder of perimeter $n$ with no seam (periodic boundary condition). If we iterate $n-1$ times the procedure used in obtaining (110), we obtain

$$
\begin{aligned}
& \operatorname{Tr}\left[Y_{k_{1}}^{\left(c_{1}, s_{1}\right)} Y_{k_{2}}^{\left(c_{2}, s_{2}\right)} \cdots Y_{k_{n}}^{\left(c_{n}, s_{n}\right)} E^{L-\sum_{j=1}^{N}\left(s_{j}-1\right)} \Omega_{P}\right]=e^{i n k_{j}\left[L-\sum_{j=1}^{N}\left(s_{j}-1\right)\right]} e^{i P \sum_{j=1}^{n}\left(s_{j}-1\right)} \\
& \sum_{c_{1}^{\prime}, \ldots, c_{n}^{\prime}}<c_{1}, \ldots, c_{n}\left|\tilde{\mathcal{T}}^{n}\right| c_{1}^{\prime}, \ldots, c_{n}^{\prime}>\operatorname{Tr}\left[Y_{k_{1}}^{\left(c_{1}^{\prime}, s_{1}\right)} \cdots Y_{k_{n}}^{\left(c_{n}^{\prime}, s_{n}\right)} E^{L-\sum_{j=1}^{N}\left(s_{j}-1\right)} \Omega_{P}\right] .
\end{aligned}
$$

Consequently, the spectral parameters $\left\{k_{j}\right\}$ should satisfy

$$
e^{-i k_{j}\left(L+n-\sum_{i=1}^{n} s_{i}\right)}=e^{i \frac{2 \pi}{n} r} e^{i P(\bar{s}-1)} \tilde{\Lambda}\left(k_{j},\left\{k_{l}\right\}\right) ; \quad j=1, \ldots, n ; \quad r=0,1, \ldots, n-1,
$$

where $\tilde{\Lambda}\left(k_{j},\left\{k_{l}\right\}\right)$ is an eigenvalue of the transfer matrix $\tilde{\mathcal{T}}$ given in (111), and $\bar{s}=\sum_{j=1}^{n} \frac{s_{j}}{n}$ is the average size of the particles. The eigenvalues $\tilde{\Lambda}\left(k_{j},\left\{k_{l}\right\}\right)$ can be obtained from the diagonalization of (111) through the coordinate Bethe ansatz and they are given by Eqs. (67) and (70) with $\Phi_{\alpha}=1$ [19]. We finally have the conditions that fix the spectral parameters of this general problem,

$$
\begin{aligned}
& e^{-i k_{j}\left[L+n-\sum_{j=1}^{n} s_{j}\right]}=(-1)^{n-1} e^{i \frac{2 \pi}{n} r} e^{i P(\bar{s}-1)} \prod_{j^{\prime}=1}^{n} \frac{\epsilon_{+}+\epsilon_{-} e^{i\left(k_{j}+k_{j^{\prime}}\right)}-e^{i k_{j}}}{\epsilon_{+}+\epsilon_{-} e^{i\left(k_{j}+k_{j^{\prime}}\right)}-e^{i k_{j^{\prime}}}} \\
& \times \prod_{l=1}^{m_{1}} \frac{\epsilon_{+}\left(e^{i k_{l}^{(1)}}-e^{i k_{j}}\right)}{\epsilon_{+}+\epsilon_{-} e^{i\left(k_{l}^{(1)}+k_{j}\right)}-e^{i k_{j}}}, \quad j=1,2, \ldots, n,
\end{aligned}
$$

where the auxiliary complex parameters $\left\{k_{j}^{(l)}, l=0, \ldots, N-1 ; j=1, \ldots, m_{l}\right\}$ are fixed by the equations

$$
\begin{gathered}
\prod_{\beta=1}^{m_{l}} \frac{\epsilon_{+}\left(e^{i k_{\alpha}^{(l+1)}}-e^{i k_{\beta}^{(l)}}\right)}{\epsilon_{+}+\epsilon_{-} e^{i\left(k_{\alpha}^{(l+1)}+k_{\beta}^{(l)}\right)}-e^{i k_{\beta}^{(l)}}}=(-1)^{m_{l+1}} \prod_{\delta=1}^{m_{l+2}} \frac{\epsilon_{+}\left(e^{i k_{\delta}^{(l+2)}}-e^{i k_{\alpha}^{(l+1)}}\right)}{\epsilon_{+}+\epsilon_{-} e^{i\left(k_{\delta}^{(l+2)}+k_{\alpha}^{(l+1)}\right)}-e^{i k_{\alpha}^{(l+1)}}} \\
\times \prod_{\alpha^{\prime}=1\left(\alpha^{\prime} \neq \alpha\right)}^{m_{l+1}} \frac{\epsilon_{+}+\epsilon_{-} e^{i\left(k_{\alpha}^{(l+1)}+k_{\alpha^{\prime}}^{(l+1)}\right)}-e^{i k_{\alpha}^{(l+1)}}}{\epsilon_{+}+\epsilon_{-} e^{i\left(k_{\alpha}^{(l+1)}+k_{\alpha^{\prime}}^{(l+1)}\right)}-e^{i k_{\alpha^{\prime}}^{(l+1)}}}, \quad l=0,1, \ldots, N-2 ; \quad \alpha=1, \ldots, m_{l},
\end{gathered}
$$

where $n_{j}(j=1, \ldots, N)$, as before, is the number of particles on class $j$ and $m_{l}=\sum_{j=1}^{N-l} n_{j}, \quad l=0, \ldots, N ; m_{0}=$ $n, m_{N}=0$, and $k_{j}^{(0)}=k_{j}$. The energies and momentum are given in terms of $\left\{k_{j}\right\}$ by (95). We can see from (114) and (115), since for each value of $r(r=0,1, \ldots, n-1)$ we have distinct solutions, that the number of solutions is higher than that previously obtained. This should be expected since the particles now are completely distinguishable.

\section{Conclusions and generalizations}

We have shown that all the exact results derived for the asymmetric exclusion problem and generalizations through the coordinate Bethe ansatz can also be obtained in an el- 
egant and unified view by an appropriate matrix product ansatz. According to this ansatz, the amplitudes of the eigenfunctions of the associated Hamiltonian are given by traces of a product of matrices. The algebraic properties of the matrices appearing in the ansatz are fixed by the eigenvalue equation of the Hamiltonian. The existence of a well defined ratio among the several amplitudes of any eigenfunction implies the associativity of the algebra ruling the matrices defining the ansatz. In the case where we have more than a single kind of particle the condition of associativity of the algebra (see (96)) coincides with the YangBaxter relations [42, 21]. Once the algebraic relations of the matrices are fixed the eigenfunctions we obtain coincide with those obtained through the coordinate Bethe ansatz. As an example, see (45) for the case of diffusion of one kind of particle with a fixed size $s$.

Differently from the Bethe ansatz solutions presented in [15], [18] and [19] the matrix product ansatz we formulate allow us to treat in a unified way the hard-core exclusion effects produced by the size of the particles. This virtue allowed a simple derivation of the quite complicated problem (see (114) and (115) in section 5) where we have $N$ types of particles hierarchically ordered, but each particle being distinguishable and with a given specified size. The corresponding calculation through the coordinate Bethe ansatz is rather difficult.

The extension of the solution presented in section 5 for the cases where the molecules are allowed to have a zero size is immediate and follows the same reasoning of sections 2 and 3 . In the case of a single species of molecule we can also extend our models allowing the molecules to have negative size $(s=-1,-2, \ldots)$ as in [45]. In this case, since we do not have the interchange process, the particles have a well defined order on the lattice, apart of cyclic rotations, i. e., $\left(x_{1} \leq x_{2} \leq \cdots \leq x_{n}\right)$. A particle $i$ with negative size $s$ allows a partial break of this ordering, i. e., $\left(x_{1} \leq x_{2} \leq \cdots \leq x_{i-1}-s \leq x_{i} \leq x_{i+1} \leq \cdots \leq x_{n}\right)$.

We may also extend the matrix product ansatz presented in this paper to the cases where the lattice size has open ends [41]. In those cases, instead of the trace operation defining the amplitudes of the eigenfunction, we have a single undefined matrix product that can be fixed by a normalization of the corresponding eigenfunction.

The success of our matrix product ansatz can also be tested [41] on an enormous variety of known exactly integrable models, irrespective if the Hamiltonian is related or not to nonequilibrium stochastic models. We have shown that our matrix product ansatz can provide the exact solutions of the XXZ chain with arbitrary exclusion effects [46], the Fateev-Zamolodchikov model [47], the Izergin-Korepin model [48], the $t-J$ model [49], the Hubbard model [50] as well as the generalized integrable models presented in [51] and [52]. In conclusion, our results suggest the conjecture that all exact integrable models may have their eigenfunctions given by an appropriate matrix product ansatz.

\section{Acknowledgements}

This work has been partly supported by FAPESP, CNPq and CAPES (Brazilian agencies).

\section{References}

[1] A. A. Lushnikov, Zh. Éksp. Teor. Fiz. 91, 1376 (1986) [Sov. Phys. JETP 64, 811 (1986)], Phys. Lett. A 120, 135 (1987).

[2] G. M. Schütz, J. Stat. Phys. 71, 471 (1993).

[3] F. C. Alcaraz, M. Droz, M. Henkel, and V. Rittenberg, Ann. Phys. (N.Y.) 230, 250 (1994).

[4] F. C. Alcaraz and V. Rittenberg, Phys. Lett. B 324, 377 (1993).

[5] F. C. Alcaraz, Int. J. Mod. Phys. B bf 8, 3449 (1994).

[6] M. D. Grynberg and R. B. Stinchcombe, Phys. Rev. Lett. 74, 1242 (1995).

[7] K. Krebs, M. P. Pfannmüller, B. Wehefritz, and H. Henrichsen, J. Stat. Phys. 78, 1429 (1995).

[8] J. E. Santos, G. M. Schütz, and R. B. Stinchcombe, J. Chem. Phys. 105, 2399 (1996).

[9] M. J. de Oliveira, T. Tomé, and R. Dickman, Phys. Rev. A 46, 6294 (1992).

[10] L. H. Gwa and H. Spohn, Phys. Rev. Lett. 68, 725 (1992), Phys. Rev. A 46, 844 (1992).

[11] D. Kim, Phys. Rev. E 52, 3512 (1995).

[12] D. Kim, J. Phys. A 30, 3817 (1997).

[13] F. C. Alcaraz, S. Dasmahapatra and V. Rittenberg, J. Phys. A. 31, 845 (1998).

[14] T. Sasamoto and M. Wadati, J. Phys. A 31, 6057 (1998).

[15] F. C. Alcaraz and R. Z. Bariev, Phys. Rev. E 60, 79 (1999).

[16] B. Derrida, Physics Reports 301, 65 (1998).

[17] T. M. Ligget, Stochastic Interacting Systems: Contact, Voter and Exclusion Process (Springer Verlag, 1999).

[18] F. C. Alcaraz and R. Z. Bariev, Braz. J. Phys. 30, 13 (2000).

[19] F. C. Alcaraz and R. Z. Bariev, Braz. J. Phys. 30, 655 (2000).

[20] G. M. Schütz, Integrable Stochastic Many-body Systems in "Phase Transition and Critical Phenomena", vol. 19, Eds. C. Domb and J. L. Lebowitz (Academic, London, 2000).

[21] R. J. Baxter, Exactly solved models in statistical mechanics (Academic Press, New York, 1982).

[22] V. E. Korepin, A. G .Izergin and N. M. Bogoliubov, Quantum Inverse Scattering Method, Correlation Functions and Algebraic Bethe Ansatz (Cambridge University Press, Cambridge, 1992).

[23] F. H. L. Essler and V. E. Korepin, Exactly Solvable Models of Strongly Correlated Electrons (World Scientific, Singapore, 1994).

[24] P. Schlottmann, Int. J. Mod. Physics B, 11, 355 (1997).

[25] C. Boldrighini, G. Cosimi, S. Frigio, and G. Nuñes, J. Stat. Phys. 55, 611 (1989).

[26] P. A. Ferrari, C. Kipnis and E. Saada, Ann. Prob. 19, 226 (1991).

[27] P. A. Ferrari, Prob. Theory Relat. Fields 91, 81 (1992).

[28] B. Sutherland, Phys. Rev. B 12, 3795 (1975).

[29] J. H. H. Perk and C. L. Schultz, Phys. Lett. A 84, 407 (1981).

[30] J. Krug and H. Spohn, Solids Far From Equilibrium Eds. C. Godrèche (cambridge University Press, Cambridge, 1991). 
[31] B. Derrida, M. R. Evans, V. Hakim and V. Pasquier, J. Phys. A 26, 1493 (1993).

[32] B. Derrida, E. Domany and D. Mukamel, J. Stat. Phys. 69, 667 (1992)

[33] R. Domany and E. Schütz, J. Stat. Phys. 72, 277 (1993).

[34] M. R. Evans, D. P. Foster, C. Godrèche and D. Mukamel, J. Stat. Phys. 80, 69 (1995).

[35] B. Derrida, J. L. Lebowitz and E. R. Speer, J. Stat. Phys. 89, 135 (1997).

[36] K. Nagel and M. Schreckenberg, J. Physique 2, 2221 (1992).

[37] R. B. Stinchcombe and G. M. Schütz, Phys. Rev. Lett. 75, 140 (1995); Europhys. Lettt. 29, 663 (1995).

[38] T. Sasamoto and M. Wadati, J. Phys. Soc. Japan 66, 2618 (1999)

[39] V. Popkov, M. E. Fouladvand and G. M. Schütz, J. Phys. A 35, 7187 (2002)

[40] V. Popkov and G. M. Schütz, Math. Fis. Anal. Geom. 9, 401 (2002).

[41] F. C. Alcaraz and M. J. Lazo, to be published.

[42] C. N. Yang, Phys. Rev. Lett. 19, 1312 (1968).
[43] K. Mallick, S. Mallick and N. Rajewsky, J. Phys. A 32, 48 (1999).

[44] S. V. Pokrovskii and A. M. Tsvelick, Zh. Eksp. Teor. Fiz. 93, 2232 (1987)(Sov. Phys.-JETP 66, 6 (1987)).

[45] A. A. Ferreira and F. C. Alcaraz, Phys. Rev. E 65, 052102 (2002).

[46] F. C. Alcaraz and R. Z. Bariev, in Statistical Physics in the Eve of the 21st Century, edited by M. T. Batchelor and L. T. Wille, Series on advances in Statistical Mechanics, Vol. 14 (World Scientic, Singapore, 1999), (Preprint condmat/9904042).

[47] A. B. Zamolodchikov and V. Fateev, Sov. J. Nucl. Phys. 32, 298 (1980).

[48] A. G. Izergin and V. E. Korepin, Commun. Math. Phys. 79, 303 (1981).

[49] P. Schlottmann, Phys. Rev. B 36, 5177 (1987).

[50] E. H. Lieb and F. Y. Wu, Phys. Rev. Lett. 20, 1445 (1968).

[51] F. C. Alcaraz and R. Z. Bariev, J. Phys. A 32, L483 (1999).

[52] F. C. Alcaraz and R. Z. Bariev, J. Phys. A 34, L467 (2001). 